Inácio, N., Nocete Calvo, F., Nieto Liñan, J. M. y Rodríguez Bayona, M. (2019): “Más allá de las tipologías: propuesta de organización social de la producción alfarera en Valencina de la Concepción (Sevilla)", Spal 28.1: 11-34. DOI: http://dx.doi.org/10.12795/spal.2019.i28.01

\title{
MÁS ALLÁ DE LAS TIPOLOGÍAS: PROPUESTA DE ORGANIZACIÓN SOCIAL DE LA PRODUCCIÓN ALFARERA EN VALENCINA DE LA CONCEPCIÓN (SEVILLA)
}

\section{BEYOND TYPOLOGIES: THE SOCIAL ORGANIZATION OF THE POTTERY PRODUCTION IN VALENCINA DE LA CONCEPCIÓN (SEVILLE)}

\author{
NUNO INÁCIO \\ Departamento Historia, Geografía y Antropología, Universidad de Huelva \\ Campus de El Carmen, Avda. de las Fuerzas Armadas, 21071 Huelva \\ Correo-e: nuno.inacio@dhis1.uhu.es (D) https://orcid.org/0000-0001-7152-677X \\ FRANCISCO NOCETE CALVO \\ Departamento Historia, Geografía y Antropología, Universidad de Huelva \\ Campus de El Carmen, Avda. de las Fuerzas Armadas, 21071 Huelva \\ Correo-e: nocete@uhu.es (D) https://orcid.org/0000-0001-5948-0748 \\ JOSÉ MIGUEL NIETO LIÑAN \\ Departamento de Ciencias de la Tierra \\ Campus de El Carmen, Avda, de las Fuerzas Armadas, 21071 Huelva \\ Correo-e: jmnieto@uhu.es (iD) https://orcid.org/0000-0002-0086-252X \\ MOISÉS R. BAYONA \\ Departamento Historia, Geografía y Antropología, Universidad de Huelva \\ Campus de El Carmen, Avda. de las Fuerzas Armadas, 21071 Huelva \\ Correo-e: moises.rodriguez@dhis1.uhu.es (D) https://orcid.org/0000-0003-4043-3954
}

\begin{abstract}
Resumen: El asentamiento de Valencina de la Concepción (Sevilla, España) es un referente en la arqueología prehistórica peninsular, no solo por sus grandes tumbas, sino también por su gran magnitud. En los últimos años, la investigación ha centrado su atención en el estudio de la organización técnica y social de las primeras producciones artesanales, con especial hincapié en la metalurgia y en la manufactura de objetos de marfil. A pesar de las innumerables evidencias, solo recientemente el componente cerámico ha sido objeto de un amplio estudio que ha permitido proponer un primer modelo de organización técnica y social de la producción alfarera. La caracterización arqueométrica (análisis petrográfico y químico) y morfológica de un conjunto significativo de muestras de cerámica, y desde ahí el estudio de las distintas etapas de la cadena operativa alfarera, permite plantear la hipótesis sobre la existencia de un modelo económico y social complejo, organizado alrededor de unidades supradomésticas que evidencian un cierto grado de especialización económica.

Palabras clave: Cerámica, Producción, Tecnología, Especialización artesanal, Estandarización.
\end{abstract}

Abstract: The settlement of Valencina de la Concepción (Seville, Spain) is one of the most important sites in the Iberian Prehistory, not only for its great tombs, but also due to its magnitude. In recent years, research has focused on the study of the technical and social organization of the first craft productions, with special emphasis on metallurgy and the manufacture of ivory objects. In spite of the innumerable evidences over the site, only recently the ceramic assemblage has been object of an analysis. The combination of archeometric (petrographic and chemical analysis) and morphological characterization allow us to reconstruct the different stages of the pottery production and to propose its first model of technical and social organization. The data provided by the investigation suggests the existence of a complex economic and social model, organized around supradomestic units that already show a certain degree of economic specialization.

Keywords: Ceramics, Production, Technology, Craft Specialization, Standardization. 


\section{INTRODUCCIÓN}

La historiografía arqueológica en el Suroeste de la península ibérica fue dominada hasta los inicios del siglo XXI por la tradición histórico-cultural, cuyo concepto normativo de cultura estuvo anclado a las explicaciones basadas en el axioma de los fósiles-directores y de sus paralelos, los inventarios descriptivos de objetos descontextualizados, a menudo evaluados por sus hallazgos superficiales, sin mediación de intervención arqueológica/científica alguna (Orihuela 1999). En este sentido, la llamada "cultura material" adquirió una relevancia trascendental, hasta el punto de transformarse en el objeto y objetivo teórico del estudio arqueológico. Desde este marco, la investigación arqueológica hizo, más bien, hincapié en las tipologías y los paralelos, descuidando sobremanera la incorporación de nuevos campos de análisis que permitiesen la formulación de novedosas y significativas preguntas. La "cultura material" como objeto de análisis y más específicamente la cerámica, de lejos el registro arqueológico con mayor presencia en los contextos arqueológicos, fue presentada como el fósil director más verosímil, eligiendo su forma y decoración como impulsores de un discurso heurístico basado en la descripción, en los paralelos y en la seriación cronológica y cultural. De este modo, la cerámica fue utilizada como principal referente arqueológico para la caracterización de horizontes culturales y cronológicos.

Ante esta situación, se hacía necesario plantear no solo nuevas preguntas, sino que estas estuvieran apoyadas por registros actualizados que incluyesen nuevos niveles de evaluación empírica, obtenidos desde proyectos sistemáticos de investigación cuyos objetivos fuesen la explicación de las condiciones materiales de la vida humana (producción, distribución y consumo). Para ello, se procedió al desarrollo de un programa de trabajo aplicado a uno de los principales referentes de la prehistoria peninsular -Valencina de la Concepciónque, por su registro, magnitud y expresión, ofrecía la posibilidad de evaluar la organización técnica y social de la actividad alfarera, permitiendo, dadas las características de sus contextos, planificar una línea de trabajo que cubriese las carencias y las necesidades estructurales en el ámbito espacial y cronológico donde se inserta.

El asentamiento prehistórico de Valencina de la Concepción se implanta en una amplia meseta con ligero declive hacia el sureste, limitado al norte por los cerros que suponen su máxima altitud y por las escarpas enmarcadas por cañadas más o menos profundas que recortan toda la vertiente $\mathrm{y}$, al sur, por pequeñas lomas que marcan el paisaje. Desde el punto de vista geológico, el entorno más inmediato de Valencina de la Concepción se encuentra dominado por formaciones sedimentarias terciarias, con excepción de los rellenos cuaternarios documentados en los terrenos bajos y asociados al río Guadalquivir y a sus afluentes principales, como la ribera de Huelva y el Guadiamar. Se trata, a grandes rasgos, de margas, arcillas más o menos carbonatadas, arenas y limos, con una composición mineralógica global de cuarzo, calcita, dolomita, feldespatos y filosilicatos (Galán y Pérez 1989).

No obstante, hace 5000 años el entorno de Valencina de la Concepción mostraba un paisaje natural diferente del actual. Los estudios sedimentológicos desarrollados en los últimos años con el objeto de delimitar la línea de costa alcanzada por la Transgresión Flandriense (Arteaga y Roos 1995; Arteaga et al. 2016; Barragán 2016; Borja 2013), han señalado que su estrategia locacional estuvo potenciada por la proximidad a un amplio estuario donde desembocaba el Guadalquivir, conformando una línea de costa profusamente recortada por acantilados (fig. 1). Alrededor de este ambiente marino, en los puntos más elevados, se distribuye una amplia red territorial de asentamientos del III milenio a.n.e., como el denominado yacimiento del Parque de Miraflores (Lara et al. 2006) o el ubicado en la Universidad Laboral de Sevilla (Fernández Gómez y Alonso 1985), donde Valencina de la Concepción parece emerger como principal centro de poder regional (Nocete 2001 y 2014).

Las características del conjunto prehistórico de Valencina de la Concepción le conceden un gran protagonismo en el entramado social que se desarrolla en el Sur de la península ibérica desde la segunda mitad del IV hasta finales del III milenio a.n.e. La ordenación territorial del bajo Guadalquivir durante la primera mitad del III milenio a.n.e. se expresa desde un armazón social complejo que alcanzará su máxima expresión alrededor del 2500 a.n.e. Por esas fechas, según las dataciones de radiocarbono actualmente disponibles, Valencina de la Concepción representaba la cabeza de un territorio que controlaba toda la cuenca baja del Guadalquivir y los territorios periféricos, convirtiéndole en un centro de poder (Costa Caramé et al. 2010; García Sanjuán 2013; García Sanjuán y Murillo-Barroso 2013; Nocete 2001 y 2014; Nocete et al. 2008, 2011 y 2014; Pajuelo y López 2001) y un espacio de agregación poblacional (Garcia Sanjuán et al. 2017).

Su proximidad a los terrenos fértiles de la campiña, la ubicación junto al antiguo estuario del Guadalquivir, aprovechando los recursos marinos de ese rico ecosistema (moluscos, pescado, etc.) y controlando las rutas 


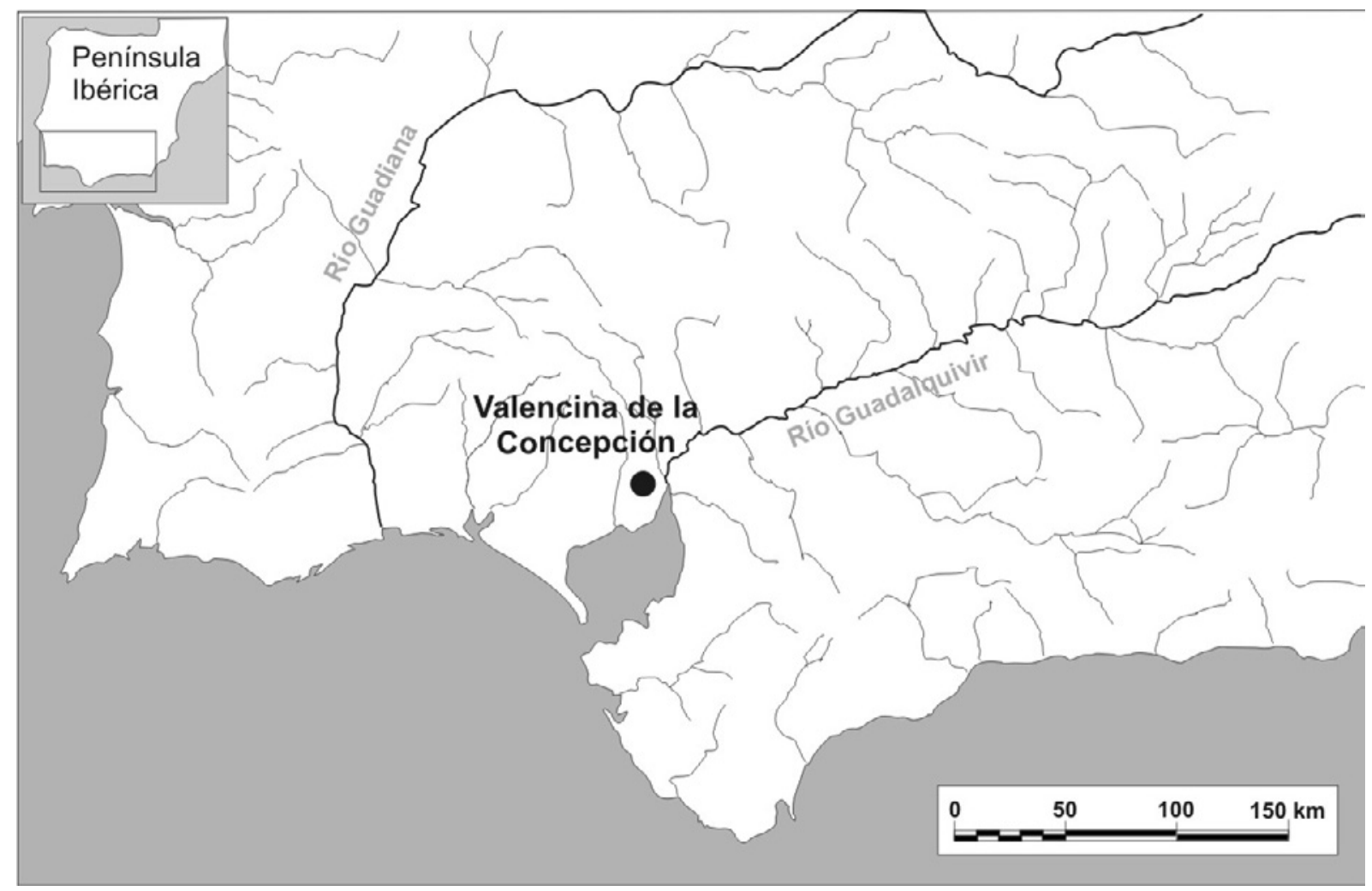

Figura 1. Localización del asentamiento prehistórico de Valencina de la Concepción.

naturales que daban acceso a los recursos líticos y minerales de la Sierra de Huelva y Sierra Morena (tufitas, dacitas, cobre, etc.), a las rutas de acceso a Los Alcores y de ahí al Subbético (caliza oolítica silicificada, cinabrio, etc.) o a las campiñas altas del Guadalquivir, señalan una ubicación estratégica que le permitía controlar una gran riqueza y diversidad de recursos que le transformaron en una "puerta de entrada" con capacidad de articular, controlar y redistribuir los recursos de origen regional (cobre, variscita, sílex, tufita, mármol, oro, cinabrio, etc.) y transcontinental (marfil), y en un centro de transformación de los mismos en productos, como evidencia la existencia de áreas artesanales dedicadas a la metalurgia o a la fabricación de productos de marfil (Nocete et al. 2014; Nocete 2014).

\section{LA CERÁMICA EN VALENCINA DE LA CONCEPCIÓN: UNA EVALUACIÓN HISTORIOGRÁFICA}

Las primeras referencias al conjunto arqueológico de Valencina de la Concepción se registran hacia finales del siglo XIX con los trabajos de Tubino (Tubino 1868) en el dolmen de La Pastora. Posteriormente le siguen, ya en el siglo $\mathrm{XX}$, el descubrimiento y excavación de otros sepulcros megalíticos, como Matarrubilla en 1917 por Hugo Obermaier (Obermaier 1919) y Ontiveros, identificado e intervenido por Carriazo en 1948 (Carriazo 1962). No es hasta la década de los 70 del siglo XX cuando se inician los trabajos de excavación en el poblado, hasta entonces únicamente referido o intuido desde la presencia de las grandes tumbas megalíticas. A partir de la década de los 80 se han desarrollado numerosas intervenciones en el casco urbano de Valencina de la Concepción y Castilleja de Guzmán, ejecutadas como excavaciones de "urgencia" o de "emergencia" para la liberación de parcelas de terreno, dando respuesta a la presión urbanística del área metropolitana de Sevilla, cuyos resultados más significativos han sido publicados a partir de 1985 en el Anuario Arqueológico de Andalucía. Ello ha generado un volumen ingente de información difícil de articular por la inexistencia de una política patrimonial global, de modelos metodológicos uniformizados de recuperación de la documentación arqueológica, de coordinación entre 
equipos y de una investigación planificada que aportase registros empíricos cualificados para su interpretación.

En 1975, Ruiz Mata publica dos artículos (Ruiz Mata 1975a y 1975b) sobre el poblado de Valencina de la Concepción basados en un pequeño corte estratigráfico de $2 \times 2 \mathrm{~m}$ realizado con el "objeto de analizar la secuencia cultural del yacimiento" (Ruiz Mata 1975a: 81). Ambos trabajos se orientaron exclusivamente a la descripción individual de dibujos de cerámica y sus resultados permitieron al autor definir una secuencia para el yacimiento de Valencina de la Concepción estructurada en dos fases culturales: una primera fase (Nivel III-Precampaniforme), con abundante cerámica común (platos, cuencos, vasos, etc.), y una segunda (Nivel II-Campaniforme), con cerámica similar a la identificada en el momento precedente pero donde fueron hallados cuatro fragmentos de cerámica decorada campaniforme, que sirvió para caracterizar esta fase en el yacimiento (Ruiz Mata 1975a).

En trabajos arqueológicos posteriores, varios autores (Fernández y Oliva 1985 y 1986; Pellicer 1979) han adoptado el desarrollo secuencial definido por Ruiz Mata (1975a, 1975b y 1983), ahora soportados por nuevos registros procedentes del poblado de los Castillejos en la Peña de los Gitanos (Arribas y Molina 1979) y por las secuencias arqueológicas del sur de Portugal (Silva y Soares 1976-1977; Silva y Soares 1981), que han permitido un mayor acierto en los criterios de periodización tipológica basada en los conjuntos ceramológicos, mediante la adopción de un esquema trifásico donde el primer momento de transición, entre el Neolítico Final y el Calcolítico Inicial, sería definido por las fuentes o cazuelas carenadas; el Calcolítico Pleno, por los platos y fuentes de borde almendrado y un tercer momento, caracterizado por la presencia de cerámica campaniforme.

A pesar de los tímidos intentos por enfatizar la importancia de la magnitud del yacimiento, todavía a finales de la década de los 80 se discutía su desarrollo interno y la adscripción cronológica y cultural con base en la presencia y/o ausencia de determinados ítems cerámicos (Caro 1989; Martín de la Cruz y Miranda 1988). No obstante, a partir de los 90, se observa un abandono paulatino del discurso normativo basado en la descripción tipológica y en la búsqueda de paralelismos formales y culturales. Los informes que se publican sobre los trabajos arqueológicos desarrollados en diversas áreas de la población homónima (ver Anuarios Arqueológicos de Andalucía) son muy escuetos en la descripción de la cultura material, relegando a los apartados de conclusiones una valoración cronológica basada en la definición bifásica (precampaniforme versus campaniforme) o, en los casos más ambiciosos, trifásica (Calcolítico Inicial, Calcolítico Pleno y Campaniforme), teniendo en cuenta la presencia o ausencia de algunos ítems en la cultura material, principalmente los fósiles directores cerámicos.

No obstante, y pese a todos los condicionamientos de la arqueología de urgencia, se han realizado algunos estudios, aunque puntuales, sobre ciertos elementos del registro arqueológico. A pesar de algunas aportaciones, incluso procedentes del análisis arqueométrico de cerámica (González Vílchez 1982; González Vílchez et al. 1999), la gran excepción parece haber sido la componente alfarera. Prueba de ello es que la reciente publicación de un volumen monográfico sobre la tutela e investigación del asentamiento prehistórico de Valencina de la Concepción, a pesar de ser un compendio global de la información disponible sobre este yacimiento, paradójicamente, no incluye ningún capítulo dedicado a la cerámica, precisamente el paradigma sobre el que han girado gran parte de las propuestas comprensivas sobre este importante sitio arqueológico (García-Sanjuán et al. 2013).

\section{LA NECESIDAD DE NUEVAS PREGUNTAS}

Una de las cuestiones centrales a la hora de definir un modelo de organización de la producción alfarera en Valencina de la Concepción es precisamente evaluar el grado de especialización que esta actividad artesanal ha alcanzado durante el III milenio a.n.e.

En las últimas décadas, la investigación arqueológica ha redireccionado su atención hacia el análisis de las condiciones sociales de la producción, distribución y consumo, con especial hincapié en el grado de especialización de las actividades artesanales. Este es uno de los conceptos centrales hacia los que ha girado el debate teórico, ya que su surgimiento ha sido considerado una forma de comprensión de la economía política de las sociedades con una organización social compleja (Brumfield y Earle 1987a y 1987b; Clark y Parry 1990; Flannery 1968 y 1972; Peregrine 1991; Stein 1996 y 1998; Tosi 1984), aunque consideramos que la naturaleza de esta relación tiene que ser investigada, ya que no está claro si la complejidad social es una condición previa para la especialización o la especialización favorece el desarrollo de la complejidad social y política, o más bien si su relación es recíproca. En términos generales, la especialización es una forma de organización de la producción y es un parámetro fundamental del análisis de la organización 
del sistema productivo global de una sociedad. Por eso sigue siendo, hoy en día, una herramienta de análisis de los aspectos sociales de la producción y su relación mutua con la distribución y consumo, ya que su aparición introduce transformaciones que van a implicar una reorganización del modelo económico de toda una comunidad.

Aunque el tema ha sido objeto de atención desde los finales del siglo XIX, en las últimas décadas varios autores han intentado (re)definir un concepto operativo con capacidad de relacionarse con los registros materiales de las comunidades pretéritas. Para C. Costin (1991) el concepto de especialización se refiere a un sistema económico diferenciado, regularizado, permanente y probablemente institucionalizado donde las actividades económicas se desarrollan según un modelo de dependencia entre los productores, que dependen de los circuitos de distribución supradomésticos, y consumidores que dependen, a su vez, de los productores que les suministran los bienes/productos que no producen. Es decir, especialización es un modelo integrado de interdependencia socioeconómica entre productores $\mathrm{y}$ consumidores, donde «fewer people make a class of objects than use it» (Costin 2001: 276 y 2005: 1036). Esta conceptualización del fenómeno se basa en el modelo de solidaridad mecánica propuesto por Durkheim, ya que enfatiza la presencia de una relación de subordinación entre productores y consumidores y los mecanismos de distribución/intercambio existentes entre estas dos esferas del proceso económico. Otros autores, sin embargo, han adoptado una definición más amplia que rechaza este mecanismo de interdependencia (Clark 1995; Cross 1993; Sassaman 1998), enfocando la cuestión en la alienación de bienes y productos, expresado en la definición «craft specialization is production of alienable, durable goods for nondependent consumption» (Clark y Parry 1990: 297).

No obstante, otras perspectivas teóricas prefieren enfatizar el papel del factor trabajo en la definición del concepto de especialización, asumiendo la especialización económica como la inversión de trabajo y capital hacia la producción de un bien o servicio en particular, de tal manera que la producción de una persona excede su propio consumo (Alchian y Allen 1969; Blackman et al. 1993; Stein 1996; Stein y Blackman 1993), implicando, a la postre, una producción de excedentes para el intercambio (Bates y Lees 1977). Así pues, no se puede analizar la especialización económica sin enfocar la naturaleza del plustrabajo (Marx 1990), y puesto que la especialización es una forma de producción, esta debe ser analizada en el entramado de relaciones sociales que constituyen el proceso de trabajo (Cobb 2000). El surgimiento de la especialización artesanal va a generar una reorganización de las relaciones sociales de producción, de los procesos de trabajo y, a la postre, motiva la división técnica y social del trabajo y la organización espacial de la producción. La especialización económica implica un reparto de las tareas productivas, al objeto de incrementar la eficacia productiva, lo que es motivado por la necesidad de aumentar la productividad y reducir los tiempos de trabajo. La especialización supone, por tanto, organizar los agentes de la producción en distintos lugares en el proceso de trabajo para asegurar que el aprendizaje y la experiencia derivada del dominio de un segmento de la producción repercutan en una mayor eficacia del tiempo de trabajo (Castro et al. 1998: 27).

Por tanto, el inicio de la transición de una producción no especializada hacia una especializada acontece cuando el artesano empieza a producir bienes para la distribución fuera de los ámbitos domésticos (Arnold 2000: 334). En este sentido, el ámbito doméstico se ha considerado como la frontera analítica para considerar o no una actividad productiva especializada, lo que supone que en una producción especializada productores y consumidores no pertenecen al mismo ámbito doméstico (Tosi 1984: 23). Así, un bien que es producido por muchos productores en relación al número de consumidores, seguramente implicará un menor grado de especialización del trabajo y viceversa.

\section{LA NECESIDAD DE NUEVOS REGISTROS}

La dimensión espacial de Valencina de la Concepción, la complejidad de sus registros arqueológicos, las numerosas intervenciones que ha sufrido a lo largo de los últimos años y la problemática urbana del contexto empresarial donde se desarrollaron, impide que el estudio del componente ceramológico se haya realizado de forma global. Para paliar tales efectos, se han seleccionado dos actuaciones arqueológicas con el registro cronológico más extenso y que han sido objeto de un estudio más amplio: sector de Nueva Valencina (NV) y la parcela del Instituto de Enseñanza Secundaria (IES) (fig. 2).

Las intervenciones arqueológicas realizadas en el sureste del yacimiento, en una área delimitada por un sistema de fosos cuyo trazado principal (E-O) define tanto el límite sur del asentamiento como separa esta área de la del ámbito funerario, ha permitido identificar un conjunto de unidades estructurales agrupadas 


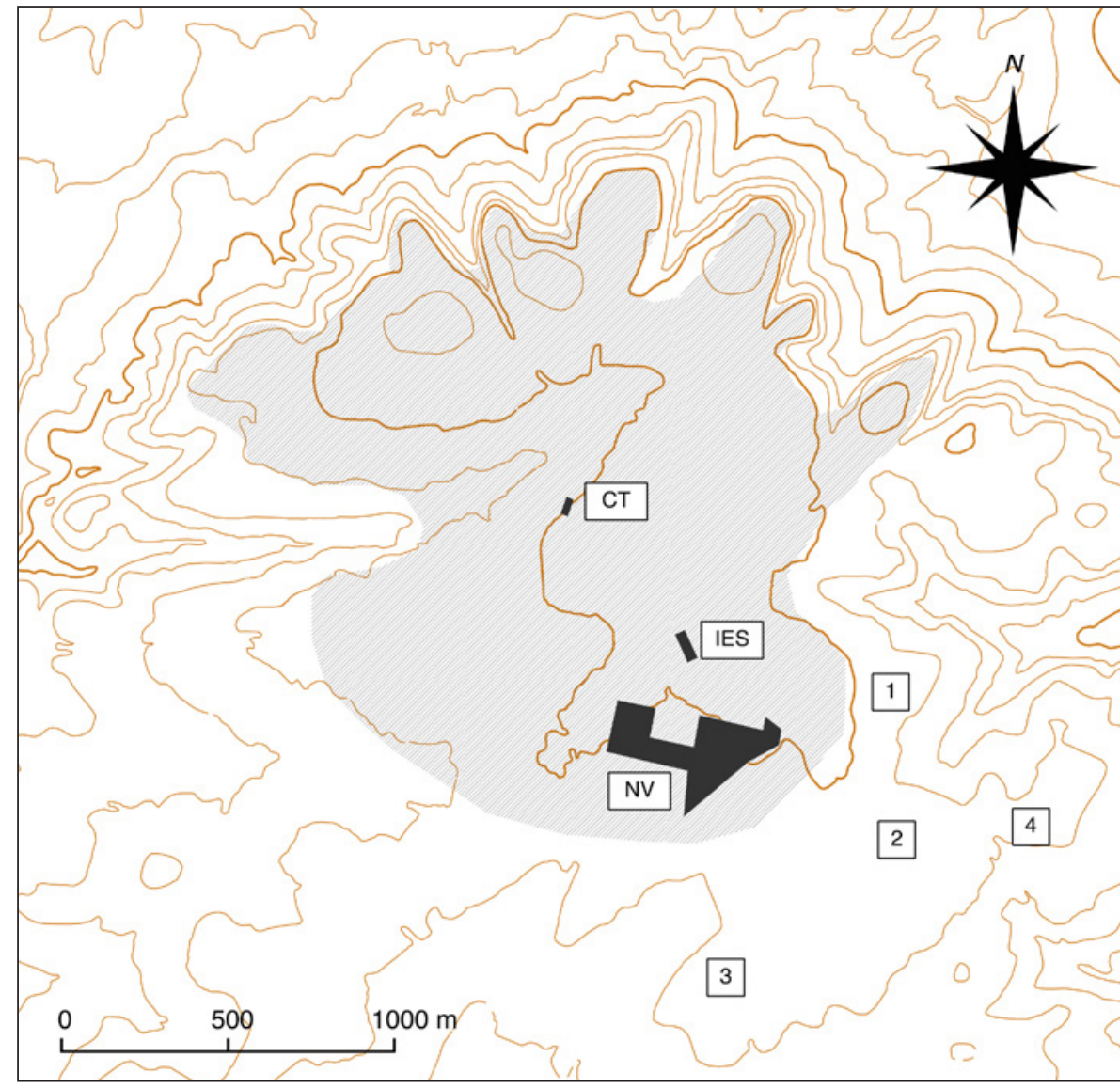

Figura 2. Ubicación de los contextos arqueológicos de Valencina de la Concepción estudiados (NV-Nueva Valencina; IES-Instituto de Enseñanza Secundaria) o referidos (CT-Calle Trabajadores) en el texto, además de las principales tumbas (1-La Pastora; 2-Ontiveros; 3-Matarrubilla; 4-Montelirio). en cuatro sectores divididos por fosos perpendiculares (N-S) de menor entidad respecto al principal. Un conjunto significativo de estas estructuras, con evidencias de fuertes termoalteraciones en sus paredes y bases, se encontraba asociada a minerales, escorias y a varias herramientas vinculadas a la actividad metalúrgica (toberas, crisoles, molinos, percutores, productos de cobre, etc.). Estos contextos definen, por tanto, una configuración espacial y económica que evidencia la actividad metalúrgica como el único sector productivo de esta área del asentamiento. Su especialización, dependencia subsistencial y delimitación espacial mediante fosos nos permiten interpretar su diseño como el de un extenso barrio artesanal (Nocete et al. 2008). La evaluación y análisis de la actividad metalúrgica en Valencina de la Concepción ha permitido la definición de un complejo sistema de producción, donde los indicadores arqueológicos señalan un nivel diferenciado y superior de desarrollo tecnológico respecto a sitios de su misma cronología.

Asimismo, a unos $200 \mathrm{~m}$ al norte se ha desarrollado una intervención arqueológica en una extensión total de $2000 \mathrm{~m}^{2}$ destinada a la construcción del aulario del nuevo instituto de enseñanza secundaria, que ha puesto en evidencia importantes contextos prehistóricos (Vargas et al. 2010). De forma general, este espacio se define por una gran complejidad estructural, donde áreas de consumo y almacenamiento doméstico comparten espacio con unidades de producción no subsistencial, algunas de las cuales resultan inéditas en el registro arqueológico de Valencina de la Concepción. Es el caso de la transformación y producción de elementos de marfil, que permite identificar un nuevo ámbito de especialización en el asentamiento (Nocete et al. 2013), ya que hasta ahora estos elementos solo habían sido identificados en los contextos ideológicos funerarios de las grandes tumbas, como en el caso del dolmen de Matarrubilla (Collantes 1968; García Sanjuán et al. 2013). Este sector del asentamiento, asociado a la transformación del marfil, permite discriminar un nuevo ámbito de especialización paralelo al de la metalurgia, ya que junto a ello, se ha identificado un posible horno de reducción de minerales de cobre. En este sentido, la información aportada por la excavación de esta área del 
asentamiento, al compartir las mismas cronologías del proceso histórico con los registros documentados en Nueva Valencina, señala la presencia de un sector artesanal especializado, concentrado y espacialmente restringido a la parte sudeste del asentamiento, donde la metalurgia adquiere una dimensión económica y social sin precedentes, en algunos puntos articulada a otros sectores artesanales.

En lo que concierne a la componente ceramológica, en términos generales, los recipientes documentados en estos contextos arqueológicos siguen los patrones morfológicos generales de la cerámica del III milenio a.n.e. del Sur de la península ibérica (fig. 3). Se trata de recipientes sin decoración con morfologías que podemos adscribir a platos, fuentes, cuencos, vasos, vasos carenados, ollas y recipientes de mayor dimensión probablemente destinados al almacenamiento/transporte (fig. 4). El examen macroscópico ha revelado que todos los recipientes han sido realizados a mano mediante la combinación de varias técnicas de manufacturación. La utilización de moldes y el posterior modelado/conformación del trozo de arcilla son la base unívoca para la gran mayoría de las cerámicas estudiadas, mientras que la técnica de rulos se ha utilizado para conformar los bordes, proporcionando distintas formas, y para levantar el cuerpo cerámico hasta la altura deseada por el ceramista. Predominan, no obstante, en todos estos contextos de Valencina de la Concepción, las formas abiertas como los platos y fuentes, a menudo con bordes engrosados, con tamaños muy estandarizados, que en algunos casos sobrepasan los $50 \mathrm{~cm}$ de diámetro. En relación a los procesos de cocción, los resultados aportados por análisis previos sugieren cocciones a baja temperatura, que en ningún caso sobrepasaron los $900^{\circ} \mathrm{C}$ (González-Vílchez et al. 1999). La mayoría de los recipientes han sido cocidos en ambientes reductores, en algunos casos con la fase final de la cocción y enfriamiento en atmósfera oxidante. En cualquier caso, la inmensa mayoría de los recipientes estudiados en este trabajo exhiben una coloración de sus superficies relativamente heterogénea, con varias manchas distribuidas de forma aleatoria por las superficies interna y externa, lo que permite plantear la hipótesis del uso de estructuras de combustión sencillas donde el proceso de cocción no es uniforme, ya que el aire y el calor circulan de forma heterogénea en su interior.

Por último, importa señalar la ausencia de cualquier evidencia de indicios de actividades alfareras (hornos, desechos, medios de producción, etc.) en los ambientes estudiados, sugiriendo que este sector artesanal se desarrolló en su exterior, definiendo, de esta manera, una separación espacial entre los contextos de producción y consumo.

\section{CARACTERIZACIÓN DE LAS MATERIAS PRIMAS Y SU PROCEDENCIA}

La caracterización de las materias primas y la determinación de su procedencia se han realizado mediante el análisis petrográfico y químico de un conjunto significativo de recipientes de cerámica: 55 del contexto de NV y 41 del IES. Para garantizar la variabilidad del muestreo y la eficiencia de los resultados, la selección de las muestras a analizar se ha realizado según los diferentes morfotipos identificados: 8 platos, 43 fuentes, 9 cuencos, 4 vasos, 5 vasos carenados, 11 ollas y 16 recipientes de grandes dimensiones (tabla 1).

Para la observación de las láminas delgadas de la cerámica se ha empleado un microscopio óptico NIKON ECLIPSE LV100POL y su caracterización ha seguido el modelo propuesto por Whitbread (1995). Los análisis químicos fueron realizados mediante las técnicas de ICP-ES (Espectrometría de Emisión con fuente de Plasma de Acoplamiento Inductivo) e ICPMS (Espectrometría de Masas con fuente de Plasma de Acoplamiento Inductivo) y los resultados han sido tratados estadísticamente mediante la transformación de las concentraciones elementales en logaritmos de razones, según Buxeda i Garrigós (1999).

En términos generales, la observación petrográfica reveló la utilización de dos tipos de arcillas para la manufactura de los recipientes de Valencina de la Concepción (producción local versus producción regional). Así pues, cerca de 9\% (n=9) de las muestras analizadas fue manufacturada con arcillas calcáreas compatibles con los depósitos cercanos de la cuenca terciaria del Guadalquivir, mientras que los restantes 91\% $(n=87)$ corresponden a recipientes producidos con arcillas secundarias procedentes de entornos geológicos dominados por rocas ígneas y metamórficas (fig. 5).

Las cerámicas carbonatadas se caracterizan por exhibir una matriz arcillosa con calcita micrítica, micas (moscovita y biotita), cuarzo y óxidos de hierro en la fracción fina. Además, la presencia de microfósiles bentónicos, esencialmente de la orden Globigerinida, es una de las características que diferencian las matrices de estas producciones (Galán y Pérez 1989). La fracción gruesa es constituida fundamentalmente por inclusiones de cuarzo (mono y policristalino), micrita, fragmentos de rocas metamórficas (filitas, esquistos, cuarcitas, etc.), fragmentos de roca plutónica de 


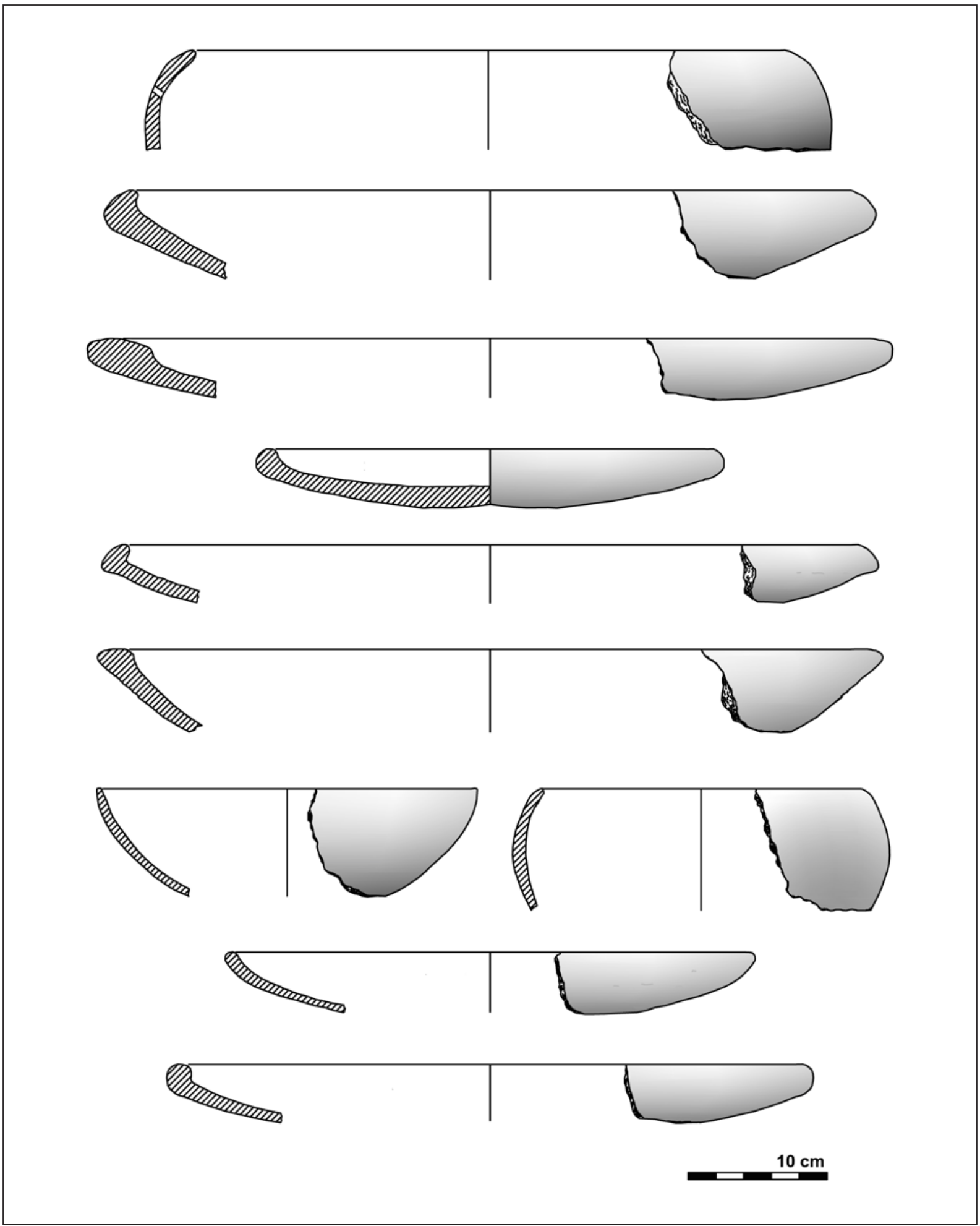

Figura 3. Recipientes de cerámica documentados en los contextos arqueológicos de NV: NFI-142, NFI-129, NFI-130, NFI-131, NFI-143, NFI-148, NFI-152, NFI-149, NFI-160 y NFI-161. 


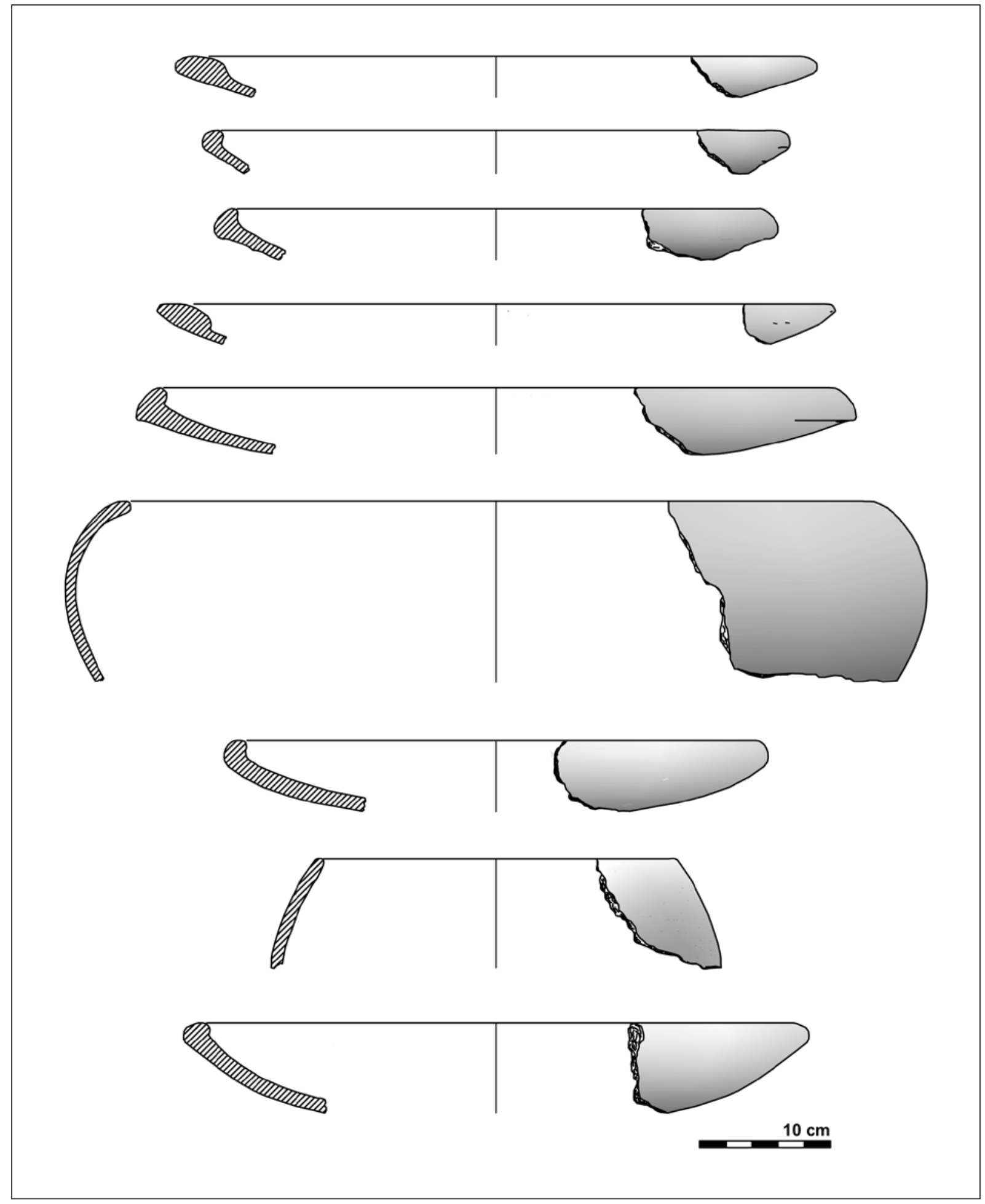

Figura 4. Recipientes de cerámica documentados en los contextos arqueológicos del IES: NFI-216, NFI-184, NFI-185, NFI-186, NFI-189, NFI-190, NFI-179, NFI-210 y NFI-211. 
Tabla 1. Recipientes seleccionados para el análisis arqueométrico.

\begin{tabular}{|c|c|c|c|c|}
\hline Sigla & Contexto & Morfotipo & Observaciones & Producción \\
\hline Val1 & NV/E182 & Morfotipo cerrado (>65 litros) & & Regional \\
\hline Val2 & NV/E182 & Fuente & Lañado & Regional \\
\hline Val3 & NV/E182 & Almacenamiento (>100 litros) & & Regional \\
\hline Val4 & NV/E182 & Vaso carenado & & Regional \\
\hline Val5 & $\mathrm{NV} / \mathrm{E} 182$ & Morfotipo cerrado perfil en "S" (>40 litros) & & Regional \\
\hline Val6 & $\mathrm{NV} / \mathrm{E} 182$ & Vaso carenado & & Regional \\
\hline Val7 & NV/E182 & Olla (>10 litros) & & Regional \\
\hline Val8 & $\mathrm{NV} / \mathrm{E} 182$ & Fuente & Retícula bruñida en el interior & Regional \\
\hline Val9 & $\mathrm{NV} / \mathrm{E} 182$ & Plato & & Regional \\
\hline Val10 & NV/E182 & Fuente & Lañado & Regional \\
\hline Val11 & $\mathrm{NV} / \mathrm{E} 182$ & Vaso carenado & & Regional \\
\hline Val12 & NV/E73/77 & Fuente & & Regional \\
\hline Val13 & NV/E73/77 & Fuente & & Regional \\
\hline Val14 & NV/E500/502 & Plato & & Regional \\
\hline Val15 & $\mathrm{NV} / \mathrm{E} 500 / 502$ & Plato & & Local \\
\hline Val16 & $\mathrm{NV} / \mathrm{E} 125$ & Morfotipo cerrado con cuello (>25 litros) & Lañado & Regional \\
\hline Val17 & $\mathrm{NV} / \mathrm{E} 125$ & Morfotipo cerrado (>30 litros) & Lañado & Regional \\
\hline Val18 & $\mathrm{NV} / \mathrm{E} 125$ & Fuente & & Regional \\
\hline Val19 & $\mathrm{NV} / \mathrm{E} 125$ & Plato & Lañado & Regional \\
\hline Val20 & $\mathrm{NV} / \mathrm{E} 125$ & Fuente & & Local \\
\hline Val21 & $\mathrm{NV} / \mathrm{E} 31$ & Cuenco & & Regional \\
\hline Val22 & NV/E31 & Fuente & & Regional \\
\hline Val23 & $\mathrm{NV} / \mathrm{E} 31$ & Fuente & & Regional \\
\hline Val24 & $\mathrm{NV} / \mathrm{E} 31$ & Olla & & Regional \\
\hline Val25 & NV/E31 & Cuenco & & Regional \\
\hline Val26 & $\mathrm{NV} / \mathrm{E} 31$ & Morfotipo cerrado (>75 litros) & & Regional \\
\hline Val27 & $\mathrm{NV} / \mathrm{E} 31$ & Cuenco & & Regional \\
\hline Val28 & $\mathrm{NV} / \mathrm{E} 31$ & Vaso carenado & & Regional \\
\hline Val29 & NV/E31 & Cuenco/Vaso & & Local \\
\hline Val30 & NV/E31 & Morfotipo cerrado (>100 litros) & & Regional \\
\hline Val31 & $\mathrm{NV} / \mathrm{E} 500 / 502$ & Morfotipo cerrado (>75 litros) & & Regional \\
\hline Val32 & NV/E500/502 & Morfotipo cerrado (>75 litros) & & Regional \\
\hline
\end{tabular}




\begin{tabular}{|c|c|c|c|c|}
\hline Sigla & Contexto & Morfotipo & Observaciones & Producción \\
\hline Val33 & NV/E500/502 & Fuente & & Regional \\
\hline Val34 & NV/E500/502 & Fuente & Lañado & Regional \\
\hline Val35 & $\mathrm{NV} / \mathrm{E} 500 / 502$ & Fuente & & Regional \\
\hline Val36 & NV/E500/502 & Fuente & & Regional \\
\hline Val37 & NV/E500/502 & Fuente & & Regional \\
\hline Val38 & NV/E191 & Vaso carenado & & Regional \\
\hline Val39 & NV/E191 & Fuente & & Regional \\
\hline Val40 & NV/E191 & Fuente & Retícula bruñida en el interior & Regional \\
\hline Val41 & $\mathrm{NV} / \mathrm{E} 191$ & Cuenco (>20 litros) & & Regional \\
\hline Val42 & $\mathrm{NV} / \mathrm{E} 73 / 77$ & Fuente & & Regional \\
\hline Val43 & $\mathrm{NV} / \mathrm{E} 73 / 77$ & Fuente & & Regional \\
\hline Val44 & $\mathrm{NV} / \mathrm{E} 73 / 77$ & Fuente & & Regional \\
\hline Val45 & NV/E73/77 & Morfotipo cerrado (>20 litros) & & Regional \\
\hline Val46 & NV/E182 & Cuenco & & Regional \\
\hline Val47 & $\mathrm{NV} / \mathrm{E} 182$ & Fuente & Lañado & Regional \\
\hline Val48 & NV/E182 & Olla perfil "S" & & Regional \\
\hline Val49 & NV/E182 & Morfotipo cerrado (>40 litros) & & Regional \\
\hline Val50 & NV/E182 & Fuente & Lañado & Regional \\
\hline Val51 & NV/E182 & Fuente & Lañado & Local \\
\hline Val52 & IES/UC402 & Fuente & & Regional \\
\hline Val53 & IES/UC402 & Fuente & Lañado & Regional \\
\hline Val54 & IES/UC402 & Fuente & & Local \\
\hline Val55 & IES/UC402 & Fuente & & Regional \\
\hline Val56 & IES/UC402 & Vaso (>18 litros) & & Regional \\
\hline Val57 & IES/UC402 & Morfotipo cerrado (>18 litros) & & Regional \\
\hline Val58 & IES/UC402 & Morfotipo cerrado (>55 litros) & & Regional \\
\hline Val59 & IES/UC99 & Fuente & & Regional \\
\hline Val60 & IES/UC99 & Fuente & & Regional \\
\hline Val61 & IES/UC99 & Fuente & & Regional \\
\hline Val62 & IES/UC99 & Morfotipo cerrado (>60 litros) & & Regional \\
\hline Val63 & IES/UC99 & Fuente & Retícula bruñida en el interior & Regional \\
\hline Val64 & IES/UC99 & Morfotipo cerrado (>55 litros) & & Regional \\
\hline Val65 & IES/UC69 & Olla & & Regional \\
\hline
\end{tabular}




\begin{tabular}{|c|c|c|c|c|}
\hline Sigla & Contexto & Morfotipo & Observaciones & Producción \\
\hline Val66 & IES/UC69 & Olla & & Regional \\
\hline Val67 & IES/UC69 & Cuenco & & Regional \\
\hline Val68 & IES/UC69 & Plato & & Regional \\
\hline Val69 & IES/UC69 & Olla & & Regional \\
\hline Val70 & IES/UC69 & Fuente & & Regional \\
\hline Val71 & IES/UC223 & Fuente & & Regional \\
\hline Val72 & IES/UC223 & Cuenco & & Regional \\
\hline Val73 & IES/UC410 & Fuente & Lañado & Regional \\
\hline Val74 & IES/UC410 & Vaso $(>20$ litros $)$ & & Regional \\
\hline Val75 & IES/UC410 & Olla & & Regional \\
\hline Val76 & IES/UC410 & Fuente & & Regional \\
\hline Val77 & IES/UC410 & Plato & & Regional \\
\hline Val78 & IES/UC50 & Vaso & & Local \\
\hline Val79 & IES/UC50 & Fuente & & Regional \\
\hline Val80 & IES/UC50 & Vaso & & Local \\
\hline Val81 & IES/UC50 & Fuente & Lañado & Regional \\
\hline Val82 & IES/UC50 & Plato & & Regional \\
\hline Val83 & IES/UC34 & Olla & & Regional \\
\hline Val84 & IES/UC34 & Olla & & Local \\
\hline Val85 & IES/UC34 & Fuente & & Regional \\
\hline Val86 & IES/UC34 & Olla & & Regional \\
\hline Val87 & IES/UC34 & Fuente & & Regional \\
\hline Val88 & IES/UC34 & Olla & & Local \\
\hline Val89 & IES/UC34 & Fuente & & Regional \\
\hline Val90 & IES/UC34 & Fuente & & Regional \\
\hline Val91 & IES/UC34 & Fuente & & Regional \\
\hline Val92 & IES/UC34 & Fuente & & Regional \\
\hline Val93 & NV/E125 & Cuenco & & Regional \\
\hline Val94 & NV/E73/77 & Plato & & Regional \\
\hline Val95 & $\mathrm{NV} / \mathrm{E} 50$ & Morfotipo cerrado ( $>25$ litros) & & Regional \\
\hline Val96 & NV/E191 & Fuente & Retícula bruñida en el interior & Regional \\
\hline
\end{tabular}


composición ácida/intermedia (granitoides con cuarzo, feldespatos y anfíboles), arenisca, micas (biotita y moscovita muy alterada) y feldespatos muy alterados. En menor proporción se han detectado inclusiones de calcarenita, arenisca, opacos, zircón y anfíboles. En este conjunto, una de las muestras se diferencia por exhibir evidencias de la utilización de un sedimento margoso, con abundantes microfósiles y concentraciones texturales, similares a las margas calcáreas que corresponden al sustrato del yacimiento.

La mayoría del muestreo estudiado presenta, sin embargo, características mineralógicas que sugieren la utilización de arcillas procedentes de entornos geológicos más distantes, en algunos casos situados a más de $10 \mathrm{~km}$ del asentamiento prehistórico. En términos generales, las muestras de este grupo exhiben una matriz no calcárea, compacta, homogénea y ópticamente activa. La variabilidad mineralógica y litológica de la fracción gruesa sugiere dos grandes áreas de aprovisionamiento compatibles con las formaciones geológicas ubicadas al norte: la Faja Pirítica Ibérica (Sáez et al. 1996; Tornos 2008) y el Batolito de la Sierra Norte de Sevilla (Rosa 1992; Rosa y Castro 2004). Así pues, además de inclusiones de cuarzo, feldespatos potásicos, plagioclasas y micas, que se encuentran presentes en diferentes proporciones en casi todas las muestras, se han detectado igualmente fragmentos de roca metamórfica (filita, cuarcita, pizarra, milonita, etc.) en un pequeño grupo de muestras, y fragmentos de roca plutónica de composición ácida e intermedia (granito, tonalita, granodiorito, etc.), además de minerales detríticos de estas rocas. La presencia de anfíboles que caracteriza gran parte de este último grupo de muestras sugiere la utilización de sedimentos arcillosos inmaduros y cercanos a las rocas plutónicas de origen.

Una aproximación a la realidad composicional ha permitido otra perspectiva sobre la variabilidad química del muestreo analizado. Así pues, en el diagrama ternario se observa la existencia de dos grupos de muestras: uno mayoritario situado en el interior del triángulo de equilibrio $\mathrm{SiO}_{2} /$ Anortita/Mulita, indicando la utilización de arcillas no calcáreas; otro en el interior del triángulo $\mathrm{SiO}_{2} /$ Anortita/Wollastonita correspondientes a muestras calcáreas. Como se ha comentado anteriormente, solo un recipiente se sitúa en el triángulo de equilibrio Anortita/Wollastonita/Gehlenita, evidenciando la utilización de arcillas margosas locales, correspondientes al propio sustrato del yacimiento (fig. 6).

El resultado del análisis estadístico de la variabilidad química se puede visualizar en el dendograma
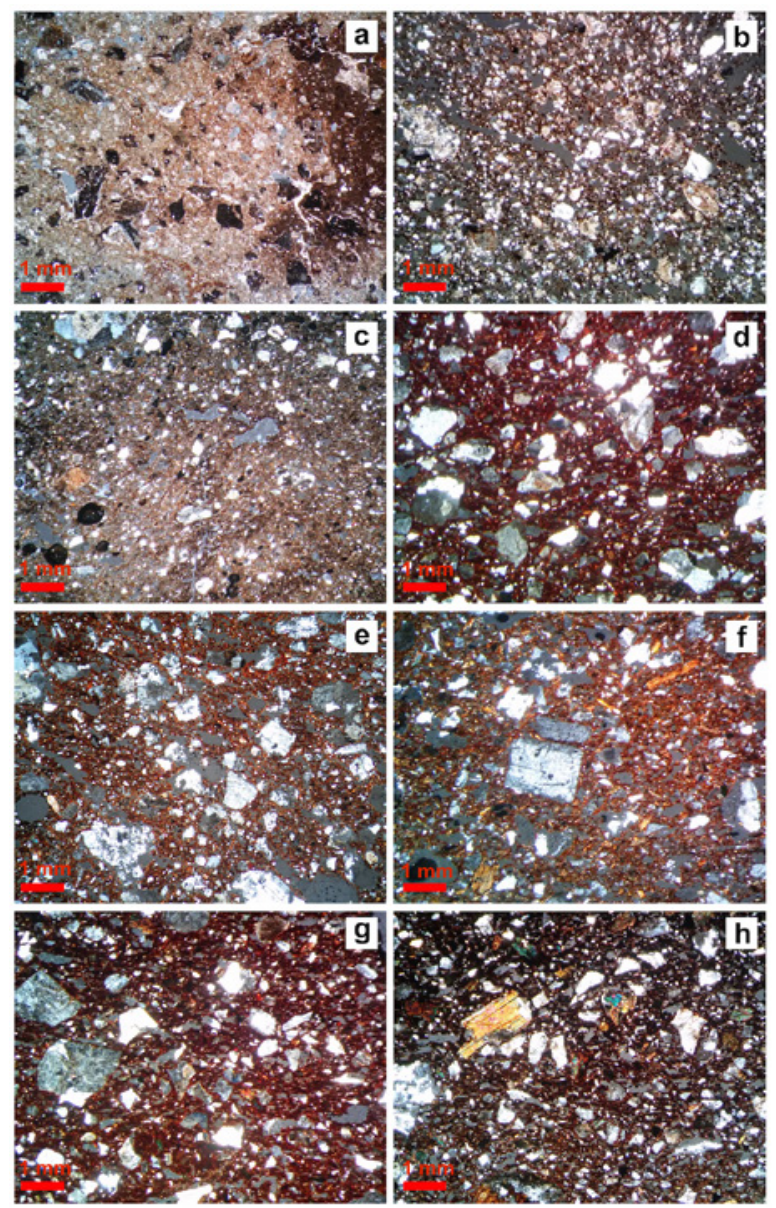

Figura 5. Microfotografías de lámina delgada obtenidas en microscopio petrográfico (nícoles cruzados). A) Matriz margosa con abundantes microfósiles y concentraciones texturales. B) Matriz arcillosa carbonatada con caliza micrítica y microfósiles. C) Matriz arcillosa carbonatada con abundantes microfósiles y inclusiones de cuarzo. D) Matriz arcillosa con fragmentos de roca plutónica ácida. E) Matriz arcillosa con fragmentos de roca plutónica ácida. F) Matriz arcillosa con inclusiones de roca plutónica intermedia, micas y anfíboles. G) Matriz arcillosa con inclusiones de roca plutónica y fracción fina con abundantes anfíboles. H) Matriz arcillosa y inclusiones de roca intermedia con plagioclasas, cuarzo y anfíboles.

extraído del análisis cluster (fig. 7). En él se pueden observar al menos cinco grupos composicionales homogéneos (tabla 2) y outliers que, de un modo general, tienen correlación con las fábricas determinadas durante la observación microscópica de las láminas delgadas. A grandes rasgos, las muestras situadas a la derecha del dendograma corresponden a los recipientes manufacturados con arcillas resultantes de la disgregación de 


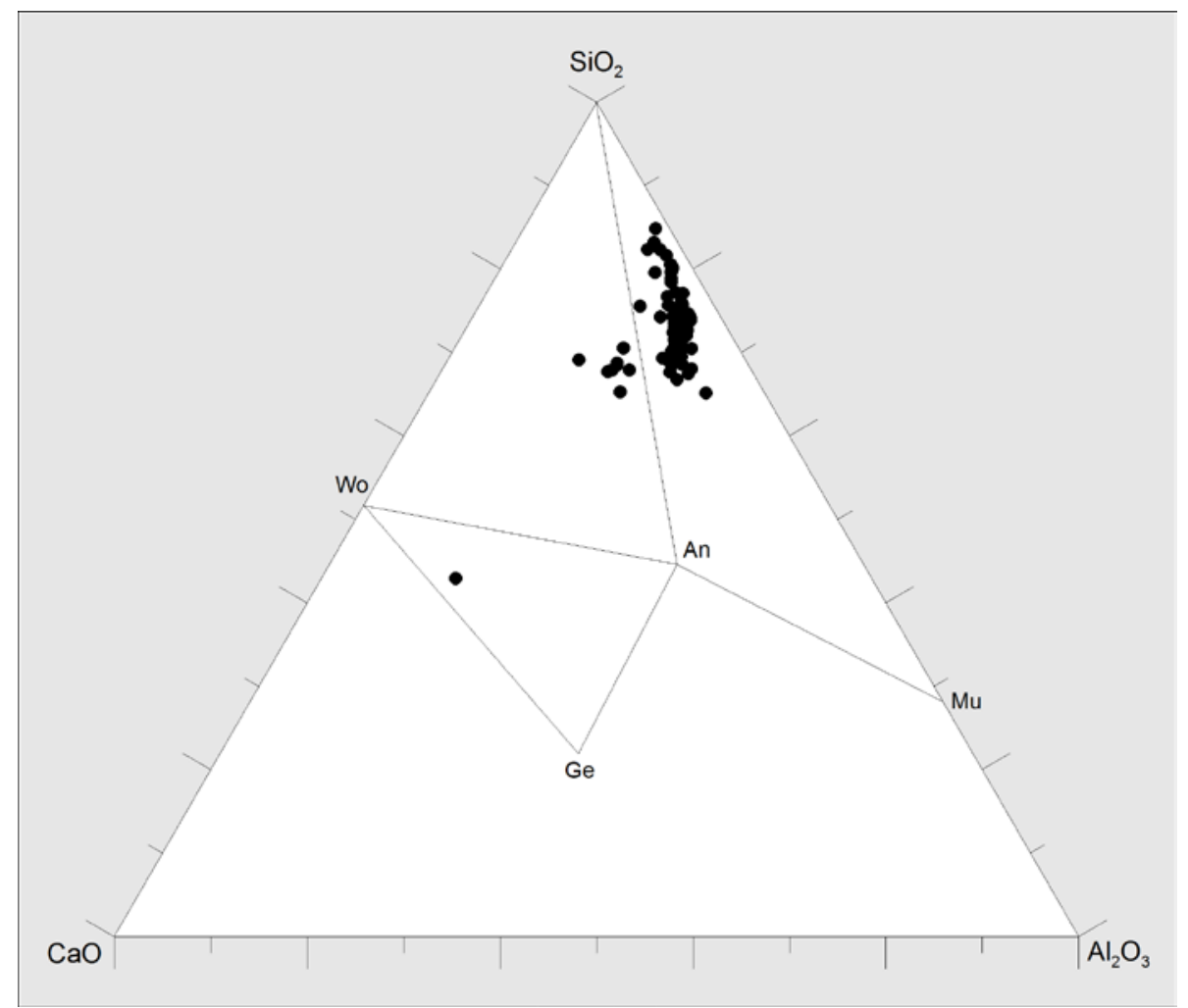

Figura 6. Representación de las muestras analizadas en el triángulo de equilibrio $\mathrm{SiO}_{2} / \mathrm{CaO} /$ $\mathrm{Al}_{2} \mathrm{O}_{3}$.

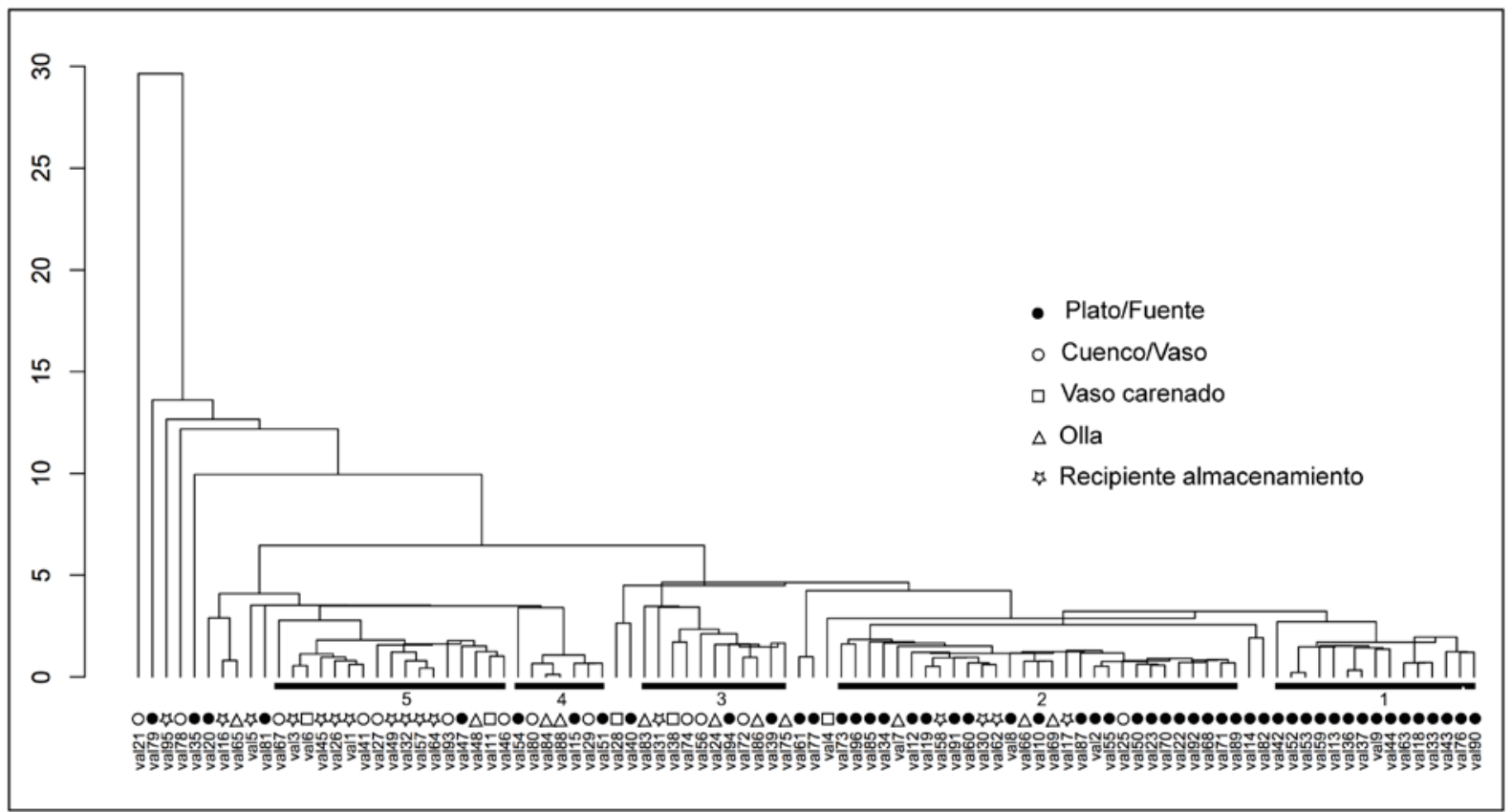

Figura 7. Dendograma resultante del análisis cluster utilizando la distancia euclidea al cuadrado como método de similitud y el algoritmo centroid como método de agrupación. 
Tabla 2. Media y desviación estándar de los grupos químicos definidos durante el análisis estadístico.

\begin{tabular}{|c|c|c|c|c|c|}
\hline & 1 & 2 & 3 & 4 & 5 \\
\hline $\mathrm{SiO} 2(\%)$ & $54,07 \pm 2,11$ & $56,85 \pm 2,33$ & $54,02 \pm 3,21$ & $50,41 \pm 1,68$ & $63,51 \pm 4,50$ \\
\hline $\mathrm{A} 12 \mathrm{O} 3(\%)$ & $17,78 \pm 0,71$ & $17,33 \pm 0,73$ & $16,44 \pm 1,18$ & $13,19 \pm 0,65$ & $15,05 \pm 2,09$ \\
\hline $\mathrm{Fe} 2 \mathrm{O} 3(\%)$ & $8,75 \pm 0,75$ & $7,53 \pm 0,76$ & $7,75 \pm 0,54$ & $5,59 \pm 0,28$ & $5,76 \pm 1,20$ \\
\hline $\mathrm{MgO}(\%)$ & $2,27 \pm 0,47$ & $1,95 \pm 0,44$ & $3,21 \pm 0,73$ & $1,70 \pm 0,43$ & $0,95 \pm 0,29$ \\
\hline $\mathrm{CaO}(\%)$ & $4,83 \pm 0,54$ & $3,79 \pm 0,65$ & $5,08 \pm 2,23$ & $9,94 \pm 0,81$ & $2,27 \pm 0,55$ \\
\hline $\mathrm{Na} 2 \mathrm{O}(\%)$ & $2,46 \pm 0,54$ & $1,68 \pm 0,41$ & $1,59 \pm 0,42$ & $0,83 \pm 0,34$ & $0,94 \pm 0,40$ \\
\hline K2O (\%) & $0,59 \pm 0,22$ & $0,86 \pm 0,23$ & $0,89 \pm 0,15$ & $1,53 \pm 0,29$ & $1,74 \pm 0,25$ \\
\hline $\mathrm{TiO} 2(\%)$ & $1,26 \pm 0,24$ & $0,99 \pm 0,23$ & $0,97 \pm 0,16$ & $0,73 \pm 0,09$ & $0,73 \pm 0,16$ \\
\hline P2O5 (\%) & $0,22 \pm 0,06$ & $0,20 \pm 0,10$ & $0,22 \pm 0,07$ & $0,28 \pm 0,05$ & $0,22 \pm 0,11$ \\
\hline $\mathrm{MnO}(\%)$ & $0,12 \pm 0,02$ & $0,09 \pm 0,02$ & $0,10 \pm 0,02$ & $0,06 \pm 0,01$ & $0,06 \pm 0,03$ \\
\hline $\mathrm{Cr} 2 \mathrm{O} 3(\%)$ & $0,01 \pm 0,00$ & $0,01 \pm 0,00$ & $0,02 \pm 0,01$ & $0,01 \pm 0,00$ & $0,01 \pm 0,00$ \\
\hline As (ppm) & $14,07 \pm 19,86$ & $18,00 \pm 21,26$ & $7,22 \pm 3,00$ & $23,98 \pm 18,97$ & $55,32 \pm 69,85$ \\
\hline $\mathrm{Ba}(\mathrm{ppm})$ & $404,07 \pm 45,08$ & $530,21 \pm 66,57$ & $487,00 \pm 73,21$ & $609,67 \pm 91,09$ & $648,94 \pm 114,83$ \\
\hline Co (ppm) & $18,99 \pm 3,72$ & $16,94 \pm 2,84$ & $25,10 \pm 4,19$ & $12,25 \pm 1,54$ & $12,04 \pm 2,53$ \\
\hline Cs (ppm) & $3,19 \pm 2,90$ & $4,50 \pm 2,90$ & $3,80 \pm 1,67$ & $5,77 \pm 3,87$ & $8,13 \pm 3,39$ \\
\hline $\mathrm{Cu}(\mathrm{ppm})$ & $31,52 \pm 24,45$ & $51,31 \pm 35,14$ & $55,31 \pm 37,52$ & $285,25 \pm 550,00$ & $353,20 \pm 708,07$ \\
\hline $\mathrm{Ga}(\mathrm{ppm})$ & $19,29 \pm 0,83$ & $18,71 \pm 1,16$ & $16,46 \pm 1,24$ & $15,57 \pm 0,92$ & $16,53 \pm 2,16$ \\
\hline Hf (ppm) & $4,66 \pm 1,08$ & $4,19 \pm 0,69$ & $3,28 \pm 0,44$ & $4,63 \pm 0,57$ & $5,20 \pm 1,02$ \\
\hline $\mathrm{Nb}(\mathrm{ppm})$ & $8,61 \pm 1,25$ & $8,78 \pm 1,74$ & $7,06 \pm 1,90$ & $12,97 \pm 1,75$ & $10,98 \pm 2,06$ \\
\hline $\mathrm{Ni}(\mathrm{ppm})$ & $13,49 \pm 3,92$ & $19,91 \pm 7,71$ & $57,38 \pm 23,50$ & $30,80 \pm 7,07$ & $27,71 \pm 9,89$ \\
\hline $\mathrm{Pb}(\mathrm{ppm})$ & $4,47 \pm 1,16$ & $6,49 \pm 1,69$ & $5,58 \pm 1,81$ & $12,77 \pm 2,34$ & $12,59 \pm 3,67$ \\
\hline $\mathrm{Rb}(\mathrm{ppm})$ & $24,46 \pm 10,08$ & $38,71 \pm 11,14$ & $35,71 \pm 9,10$ & $64,27 \pm 16,19$ & $79,05 \pm 13,12$ \\
\hline $\mathrm{Sc}(\mathrm{ppm})$ & $27,07 \pm 4,08$ & $23,31 \pm 3,75$ & $24,56 \pm 3,32$ & $14,83 \pm 1,94$ & $14,31 \pm 2,52$ \\
\hline $\mathrm{Sr}(\mathrm{ppm})$ & $205,15 \pm 27,24$ & $158,93 \pm 20,97$ & $145,31 \pm 32,31$ & $239,17 \pm 28,99$ & $100,67 \pm 23,20$ \\
\hline $\mathrm{Ta}(\mathrm{ppm})$ & $0,56 \pm 0,06$ & $0,59 \pm 0,11$ & $0,50 \pm 0,09$ & $0,87 \pm 0,12$ & $0,77 \pm 0,13$ \\
\hline $\mathrm{Th}$ (ppm) & $3,41 \pm 1,04$ & $4,83 \pm 1,10$ & $4,86 \pm 1,04$ & $7,73 \pm 0,92$ & $8,78 \pm 1,69$ \\
\hline $\mathrm{U}(\mathrm{ppm})$ & $0,74 \pm 0,21$ & $0,94 \pm 0,21$ & $0,90 \pm 0,21$ & $1,78 \pm 0,37$ & $1,56 \pm 0,34$ \\
\hline $\mathrm{V}(\mathrm{ppm})$ & $192,79 \pm 29,37$ & $157,83 \pm 28,47$ & $157,22 \pm 33,10$ & $113,33 \pm 9,95$ & $118,00 \pm 33,27$ \\
\hline $\mathrm{Y}(\mathrm{ppm})$ & $25,63 \pm 3,62$ & $20,60 \pm 3,16$ & $21,44 \pm 3,62$ & $21,62 \pm 1,91$ & $23,38 \pm 4,85$ \\
\hline $\mathrm{Zn}(\mathrm{ppm})$ & $39,57 \pm 7,70$ & $45,86 \pm 7,62$ & $44,22 \pm 14,43$ & $75,50 \pm 14,64$ & $46,38 \pm 7,18$ \\
\hline $\mathrm{Zr}(\mathrm{ppm})$ & $175,30 \pm 47,41$ & $146,87 \pm 25,23$ & $115,12 \pm 19,00$ & $164,80 \pm 19,68$ & $182,65 \pm 36,04$ \\
\hline $\mathrm{La}(\mathrm{ppm})$ & $14,39 \pm 2,31$ & $16,20 \pm 2,97$ & $15,08 \pm 3,60$ & $24,05 \pm 3,59$ & $24,98 \pm 5,88$ \\
\hline $\mathrm{Ce}(\mathrm{ppm})$ & $36,78 \pm 4,92$ & $39,53 \pm 6,44$ & $34,82 \pm 8,15$ & $53,52 \pm 6,36$ & $56,09 \pm 13,04$ \\
\hline
\end{tabular}




\begin{tabular}{|l|c|c|c|c|c|}
\hline & 1 & 2 & 3 & 4 & 5 \\
\hline $\operatorname{Pr}(\mathrm{ppm})$ & $4,46 \pm 0,61$ & $4,46 \pm 0,72$ & $4,14 \pm 0,91$ & $6,03 \pm 0,78$ & $6,37 \pm 1,47$ \\
\hline $\mathrm{Nd}(\mathrm{ppm})$ & $19,27 \pm 2,71$ & $18,34 \pm 2,79$ & $17,08 \pm 3,29$ & $23,73 \pm 2,20$ & $24,66 \pm 5,76$ \\
\hline $\mathrm{Sm}(\mathrm{ppm})$ & $4,44 \pm 0,51$ & $3,88 \pm 0,56$ & $3,63 \pm 0,59$ & $4,55 \pm 0,30$ & $4,71 \pm 1,04$ \\
\hline $\mathrm{Eu}(\mathrm{ppm})$ & $1,32 \pm 0,14$ & $1,08 \pm 0,14$ & $0,99 \pm 0,07$ & $1,04 \pm 0,06$ & $1,03 \pm 0,18$ \\
\hline $\mathrm{Gd}(\mathrm{ppm})$ & $4,67 \pm 0,56$ & $3,80 \pm 0,55$ & $3,62 \pm 0,49$ & $4,04 \pm 0,25$ & $4,24 \pm 0,86$ \\
\hline $\mathrm{Tb}(\mathrm{ppm})$ & $0,82 \pm 0,10$ & $0,68 \pm 0,10$ & $0,65 \pm 0,08$ & $0,70 \pm 0,05$ & $0,73 \pm 0,15$ \\
\hline $\mathrm{Dy}(\mathrm{ppm})$ & $4,75 \pm 0,57$ & $3,85 \pm 0,55$ & $3,84 \pm 0,52$ & $3,93 \pm 0,29$ & $4,08 \pm 0,79$ \\
\hline $\mathrm{Ho}(\mathrm{ppm})$ & $0,97 \pm 0,13$ & $0,80 \pm 0,12$ & $0,79 \pm 0,12$ & $0,79 \pm 0,07$ & $0,84 \pm 0,17$ \\
\hline $\mathrm{Er}(\mathrm{ppm})$ & $2,87 \pm 0,37$ & $2,35 \pm 0,33$ & $2,34 \pm 0,42$ & $2,30 \pm 0,22$ & $2,48 \pm 0,49$ \\
\hline $\mathrm{Tm}(\mathrm{ppm})$ & $0,44 \pm 0,05$ & $0,37 \pm 0,06$ & $0,37 \pm 0,07$ & $0,36 \pm 0,04$ & $0,39 \pm 0,08$ \\
\hline $\mathrm{Yb}(\mathrm{ppm})$ & $2,75 \pm 0,33$ & $2,33 \pm 0,32$ & $2,31 \pm 0,49$ & $2,25 \pm 0,22$ & $2,46 \pm 0,43$ \\
\hline $\mathrm{Lu}(\mathrm{ppm})$ & $0,41 \pm 0,05$ & $0,36 \pm 0,05$ & $0,35 \pm 0,07$ & $0,33 \pm 0,03$ & $0,38 \pm 0,08$ \\
\hline
\end{tabular}

rocas plutónicas de composición intermedia (Grupos 1 y 2). En el centro del dendograma se ubican aquellos recipientes manufacturados con arcillas procedentes de entornos geológicos dominados por rocas plutónicas ácidas y metamórficas (Grupo 3). De igual modo, la fábrica carbonatada se individualiza en único grupo composicional (Grupo 4), con la excepción de la muestra ya referida que tiene un comportamiento como outlier.

No obstante, debe ser destacada la correlación entre algunos grupos químicos definidos en el análisis cluster y la morfología de los recipientes. Como se puede observar en la figura 7, los recipientes clasificados morfológicamente como platos y fuentes se encuentran a la derecha del dendograma. Asimismo, los recipientes de mayores dimensiones aparecen concentrados a la izquierda del dendograma. Ello sugiere que algunos recipientes de cerámica han sido manufacturados siguiendo una receta tecnológica uniforme en lo que concierne al tipo de materia prima utilizada y a su preparación, reflejándose en una composición química homogénea.

\section{UNIFORMIZACIÓN DE LAS ELECCIONES TECNOLÓGICAS}

La ausencia de inequívocos contextos de producción pone en evidencia la necesidad de desarrollar alternativas metodológicas con el objetivo de estudiar la escala, intensidad y la organización económica y social de la actividad alfarera de Valencina de la Concepción. Entre otros métodos indirectos de evaluación, la adopción de procedimientos tecnológicos complejos y la normalización de los procesos productivos suelen ser parámetros empleados en la identificación y definición de la escala de la producción.

En términos generales, estandarización se refiere al relativo grado de homogeneidad en las características formales y materiales de los recipientes de cerámica y puede considerarse como una consecuencia del aumento trabajo invertido en una actividad, ya que la repetición de las elecciones tecnológicas suele ser responsable directa de la disminución de esa variabilidad. La introducción de secuencias rutinarias en el proceso de producción no solo va a aumentar la eficiencia del trabajo sino que, además, puede tener implicaciones en la estandarización de los productos finales. En concreto, se trata de lograr una disminución de la variabilidad de los factores que intervienen en el proceso productivo a través de la homogeneización de los procesos, rutinas e instrumentos de trabajo, de los espacios de producción y de los recursos utilizados.

Con base en observaciones etnográficas, varios autores han sugerido la existencia de relación entre la especialización artesanal y el grado de estandarización material y formal de los recipientes de cerámica, ya que se supone que la habilidad y destreza de los artesanos se desarrollan con la repetición de los procesos de trabajo (Arnold y Nieves 1992; Blackman et al. 1993; Costin 
1991 y 2000; Costin y Hagstrum 1995; Feinman et al. 1984; Longacre 1999; Rice 1981, 1987 y 1991; Sinopoli 1988).

La especialización se encuentra asociada al intento de aumentar la productividad y la eficacia de la tarea productiva, mediante, por ejemplo, la disminución de la variabilidad de los factores que intervienen en el proceso productivo. Ello supone que los conjuntos con una elevada estandarización formal y/o tecnológica se encuentran asociados a una única unidad o a un número limitado de unidades de producción con procesos de trabajo uniformes, organizados y simplificados (Costin 1991: 33). Por el contrario, conjuntos más heterogéneos suponen la existencia de un mayor número de unidades productivas, mayor heterogeneidad del proceso de trabajo y menor precisión y control sobre las características del producto final.

El concepto de estandarización puede ser empleado en el estudio de las distintas etapas de la cadena operativa y puede materializarse en dos ámbitos: la estandarización morfométrica y la estandarización material.

La estandarización morfométrica (forma y tamaño) resulta de la utilización de procesos de trabajo rutinarios sobre todo en el modelado de las vasijas (Benco 1987 y 1988). En este sentido, se supone que la repetición de los gestos del alfarero, su experiencia y práctica constante, tienen como resultado la manufactura de recipientes más estandarizados. Así pues, la presencia de un conjunto limitado de productores (especialistas) introducirá una menor variabilidad de comportamientos idiosincrásicos que se reflejarán en una menor variabilidad de los productos manufacturados.

Por el contrario, la estandarización material resulta principalmente de los comportamientos asociados a la apropiación, aprovisionamiento y preparación de las materias primas, y se expresa en las características físicas, químicas y mineralógicas de los recipientes. La premisa básica de la estandarización material es que un conjunto cerámico producido en la misma unidad de producción es más homogéneo desde el punto de vista material que un conjunto producido en diferentes unidades. Por ejemplo, el análisis químico permite evaluar el grado de estandarización del conjunto cerámico a través del análisis de la variabilidad composicional de cada elemento químico. Si la cerámica posee valores elevados de homogeneidad en la composición química, indicia la existencia de uniformización a nivel del aprovisionamiento y de la preparación de la materia prima.

El análisis morfométrico y arqueométrico de los recipientes cerámicos procedentes de Valencina de la Concepción ha proporcionado datos e información que sugieren la existencia de un modelo de organización alternativo, que difícilmente encaja en las explicaciones que definen la alfarería, al igual que otras actividades, como un sistema de producción de nivel doméstico, de autoabastecimiento, de irrelevante nivel económico, de escaso desarrollo tecnológico y de baja capacidad productiva. En términos generales, la cerámica de Valencina de la Concepción muestra una serie de características que permiten plantear la hipótesis de un modelo de organización de la producción alfarera alrededor de unidades supra domésticas que evidencian ya un cierto grado de especialización económica, a saber:

1) El resultado del análisis químico y mineralógico señala la existencia de diferentes estrategias de aprovisionamiento de recursos arcillosos para la manufactura de las vasijas de cerámica de Valencina de la Concepción. Así, a pesar de la ubicación del asentamiento junto a potenciales fuentes de aprovisionamiento, cerca del $91 \%$ de la producción cerámica fue realizada con arcillas procedentes de entornos geológicos influenciados por rocas ígneas y metamórficas, en algunos casos ubicados a más de $10 \mathrm{~km}$ del asentamiento. La utilización de materias primas procedentes de entornos foráneos aumentaría ciertamente el tiempo y los costes de transporte y de producción. En este sentido, habría que enfatizar que estas características difícilmente se encuadran en un modelo de producción alfarero no especializado, expeditivo y subordinado a actividades productivas subsistenciales, ya que en estos casos los productores tienden a restringir su inversión de tiempo, energía y trabajo al proceso productivo.

2) Resulta especialmente importante señalar la existencia de una uniformización en los procesos de aprovisionamiento, en las estrategias de captación de materias primas y en la utilización de ciertas arcillas para la manufactura de algunos morfotipos específicos, sugiriendo así una relación entre tecnología y uso social. Esta relación demuestra una estrategia productiva diferenciada, manifestada por una correlación directa entre la materia prima y la funcionalidad de los recipientes. Estos datos indican que la producción de algunos recipientes de Valencina de la Concepción ha obedecido a una receta tecnológica concreta, planificada y estandarizada. Además, habría que señalar que el reducido índice de variabilidad química se manifiesta en la presencia de grupos composicionales con valores químicos muy homogéneos, considerados aceptables en la definición de grupos de referencia (Hein et al. 1999; Tsolakidou et al. 2002), sugiriendo la 
Tabla 3. Comparación del coeficiente de variación (C.V.) del diámetro de borde en las dos técnicas de manufactura identificadas en los recipientes tipo plato/fuentes.

\begin{tabular}{|c|c|c|c|c|c|}
\hline \multicolumn{3}{|c|}{ Técnica de Manufactura A } & \multicolumn{3}{c|}{ Técnica de Manufactura B } \\
\hline $\boldsymbol{X}$ & $\sigma$ & C.V. & $\boldsymbol{X}$ & $\sigma$ & C.V. \\
\hline 44,1 & 6,1 & 13,6 & 44,6 & 7,3 & 16,3 \\
\hline
\end{tabular}

existencia de un número limitado de unidades de producción. Por el contrario, si considerásemos la existencia en Valencina de la Concepción de un gran número de unidades productivas (individuos, talleres, etc.), sería probable una mayor variabilidad material de los productos. Si supusiésemos que la producción alfarera en Valencina de la Concepción es una actividad desarrollada alrededor de las unidades domésticas, a tenor de los registros aportados por las decenas de intervenciones arqueológicas de las últimas décadas, existirían igualmente centenares de unidades de producción alfarera. En este caso, si considerásemos que cada unidad de consumo o unidad doméstica (household) fuese, al mismo tiempo, una unidad de producción alfarera, la diversidad esperada en los parámetros tecnológicos y morfométricos del conjunto cerámico sería ciertamente más elevada que la observada, ya que dependería de las elecciones tecnológicas individuales y del habitus de una multiplicidad de productores.

3) Los recipientes más representativos en toda Valencina de la Concepción (platos y fuentes) muestran un elevado grado de estandarización morfométrica que se manifiesta principalmente en el diámetro de borde. La manufactura de estos recipientes parece haber seguido un patrón muy normalizado, que sugiere una tradición tecnológica alfarera consolidada en la comunidad. Estos índices de normalización se deben a la utilización de moldes que, por las huellas que han dejado en las superficies externas de algunos casos, corresponden probablemente a otros recipientes con formato análogo o a unos mismos moldes de cerámica manufacturados con ese propósito que han ayudado a conformar el barro. Asimismo, se han podido diferenciar dos técnicas de manufacturación (tabla 3): la primera consiste en la utilización de un molde para la conformación del recipiente, produciendo bordes con una sección semicircular simple, sin espesamiento (Técnica de Manufactura A); la segunda reúne los recipientes que han sido elaborados con ayuda de un molde y a los que posteriormente les ha sido aplicado un rollo de arcilla en todo el diámetro del recipiente para su finalización, confiriéndoles mayor resistencia mecánica y formando bordes engrosados típicos de los registros ceramológicos del III milenio a.n.e. en el Sur peninsular (Técnica de Manufactura B). Esta estandarización formal de los productos cerámicos y la utilización de medios de producción como moldes en su conformación sugiere la existencia de procesos de trabajo normalizados, adoptados con el objetivo de aumentar la producción, incrementar la calidad de los productos y disminuir el tiempo invertido en su confección.

\section{CONSUMO, DEMANDA Y RECICLAJE}

Las intervenciones arqueológicas desarrolladas en Valencina de la Concepción en las últimas décadas han puesto en evidencia la existencia de un registro arqueológico complejo, donde destacan los conjuntos inmensurables de recipientes de cerámica, a veces hallados completos, pero en la mayoría de los casos fragmentados. Ello pone de manifiesto la importancia de las vasijas de cerámica en las prácticas sociales relacionadas con los contextos sociales de preparación, almacenamiento, reparto y consumo de alimentos y bebidas. Asimismo, el tamaño del asentamiento y la concentración de registros cerámicos -en total, el peso de las vasijas de cerámica puede ascender a varias toneladas-, sugieren la existencia de una fuerte demanda social de recipientes cerámicos. Además, habría que considerar que estos recipientes de uso doméstico diario e intensivo tendrían ciertamente un tiempo de utilización limitado, ya que los índices de rotura serían elevados (comprobados por la documentación de procesos de reciclaje), lo que llevaría a una pequeña pero constante demanda que solo podría ser solventada con la intensificación y el aumento de la producción. Ello sería ciertamente suficiente para mantener un sector de la población liberada de la producción subsistencial y dedicada, aunque parcialmente, a la actividad alfarera. 
Además, la consolidación de ciertas prácticas de reciclaje y mantenimiento de recipientes en los ámbitos sociales de producción metalúrgica, principalmente en el sector de Nueva Valencina, como sugiere la elevada presencia de perforaciones (lañados) asociadas a vasijas clasificadas como los platos y fuentes -precisamente aquellos que fueron manufacturados con arcillas no locales y que muestran mayores índices de estandarización tecnológica-, señalan el desarrollo de técnicas de reparación con el objeto de incrementar la duración de estos recipientes. Esta práctica, aunque casi siempre comporta una pérdida de eficacia y robustez, no tiene necesariamente como consecuencia una menor expectativa de vida útil del recipiente. No obstante, ello sugiere la desvinculación, al menos parcial, de los procesos de producción alfarera de parte de la comunidad, llevándolos a desarrollar estrategias alternativas de mantenimiento de los recipientes de uso doméstico, ciertamente costosos de obtener.

\section{PROPUESTA DE MODELO DE ORGANIZACIÓN SOCIAL DE LA PRODUCCIÓN ALFARERA EN VALENCINA DE LA CONCEPCIÓN}

Como se ha expuesto, el análisis de la cerámica de Valencina de la Concepción muestra una lenta y paulatina tendencia hacia una mayor estandarización y especialización de los procesos de producción, consecuencia de una mayor inversión de tiempo, trabajo y recursos en las tareas productivas, lo que acabó provocando una homogeneización de los procedimientos técnicos, principalmente en los recipientes de mayor demanda. Durante la primera mitad del III milenio a.n.e. se consolidó una tradición alfarera en Valencina de la Concepción que supuso la incorporación de nuevos medios técnicos además de algunas innovaciones destacadas. Para dar respuesta al incremento de las necesidades de consumo, las unidades de producción adoptaron procesos de trabajo homogéneos que se reflejan en la selección preferencial de la materia prima, estandarización en el perfil, normalización de los procesos de manufactura y acabado de los recipientes.

Varios estudios etnográficos apoyan esta hipótesis, ya que han documentado procesos de intensificación y especialización en la manufactura de bienes utilitarios, como los procedentes de la alfarería. Así por ejemplo, en los trabajos llevados a cabo en las comunidades alfareras de Kalinga (Filipinas), M. Stark (1995) describe detalladamente los mecanismos sociales que han impulsado el desarrollo de unidades especializadas en la producción de bienes utilitarios alfareros, como alternativa estratégica de subsistencia. En Dalupa, una de las aldeas de Kalinga, esta autora señala la existencia de al menos tres categorías de mujeres alfareras: (1) alfareras que desarrollan sus actividades de forma ocasional para su propio consumo; (2) alfareras que desarrollan estas actividades como complemento a las de subsistencia; (3) alfareras que tienen en la alfarería su principal actividad económica, aunque a tiempo parcial y/o estacional. Las motivaciones económicas que han llevado a la especialización alfarera en esta comunidad han estado orientadas a su distribución fuera de los ámbitos domésticos y el intercambio.

Estos ejemplos ponen de manifiesto el desarrollo de mecanismos que favorecen la especialización en la producción de bienes utilitarios. Así pues, estas primeras formas de especialización se diferencian en muchos aspectos de las producciones especializadas de ítems de prestigio, y es por ello por lo que el modelo ahora propuesto para Valencina de la Concepción no tiene, hasta al momento, paralelo en la alfarería del III milenio en el Sur de la península ibérica.

Ello contrasta con el modelo de organización que ha sido probablemente el dominante en el III milenio a.n.e. Así por ejemplo, en Cabezo Juré, el estudio de las distintas etapas del proceso de producción sugiere que la actividad alfarera se desarrolló alrededor de las relaciones sociales de ámbito doméstico, con una producción auto-suficiente, ocasional, comunitaria y destinada a satisfacer las necesidades de aquella comunidad (Inácio, 2015).

Por otro lado, la emergencia de la cerámica campaniforme en Valencina de la Concepción supone una información añadida a la complejidad que alcanzó la actividad alfarera durante este periodo. El análisis de los contextos arqueológicos de la calle Trabajadores (fig. 2) y la asociación en un mismo contexto de un conjunto de elementos que se pueden vincular con las élites que vivieron en Valencina de la Concepción lo que convierte a los vasos campaniformes como elementos de prestigio y de legitimación ideológica de las relaciones de materialización del poder (DeMarrais et al. 1996)- sugieren la existencia de mecanismos de control de la producción y distribución, inhibiendo, por ejemplo, el desarrollo de procesos de emulación o reproducción de estas vasijas (Inácio et al. 2017).

El modelo de producción alfarera de escala supradoméstica y controlada políticamente por élites sociales mediante la centralización de la producción, parece generalizarse en el II milenio a.n.e. en el Sureste de la 
península ibérica (Aranda 2004 y 2010). Según este autor, con base en el estudio de la componente ceramológica de varios asentamientos argáricos y sus respectivas necrópolis, las producciones de este periodo siguen parámetros formales, métricos y técnicos muy estrictos y normalizados que sugieren una producción especializada, próxima a lo denominado por algunos autores como industria de tipo doméstico (Colomer 2005). Además, plantea que la manufactura de cerámicas específicamente destinadas para el ritual funerario de las élites sociales argáricas estarían ciertamente bajo el control de éstas mismas élites, de cara a inhibir la reproducción de estos elementos con un valor simbólico relevante para las comunidad y para la consolidación del poder y del estatus social, en cierta forma como continuación del papel de las cerámicas campaniformes.

A pesar de que el modelo ahora propuesto para una parte significativa de la cerámica documentada en los ámbitos sociales de Valencina de la Concepción asume una producción supradoméstica y especializada, no habría que descartar, sin embargo, la existencia de unidades productivas locales de autoabastecimiento, no especializadas, destinadas a solventar una parte del abastecimiento de recipientes de los grupos domésticos. Este modelo ocasional y poco intenso de producción se mantiene probablemente en los ámbitos más restringidos de la esfera de las comunidades domésticas, apartado aparentemente de los cánones tecnomorfológicos del modelo anterior. Esta dualidad en las formas de organización de actividades artesanales ha sido recientemente enfatizada precisamente en Valencina de la Concepción para el caso de la metalurgia, ya que paralelamente al extenso barrio artesanal identificado en el sector sureste del asentamiento (Nocete et al. 2008), evidencias arqueológicas no descartan el desarrollo de actividades metalúrgicas en el seno de ámbitos domésticos ubicados en otras áreas del poblado (Costa Caramé et al. 2010).

\section{CONCLUSIÓN}

El desarrollo de la investigación en torno a la variabilidad de conductas económicas y a los modelos que han sustentado las producciones artesanales especializadas del III milenio a.n.e. en el Sur de la península ibérica comienza ahora a exhibir las características del complejo mosaico social en el cual se encuentran insertas. La consolidación del modelo agrario y la progresiva complejidad social que se observa en el Sur de la península ibérica desde la segunda mitad del IV milenio a.n.e. estimuló el surgimiento y la afirmación de una sociedad cada vez más dividida entre productores y no productores, ambos ocupando diferentes posiciones en los procesos de producción, distribución y consumo de bienes. Así pues, no descartando el surgimiento de actividades previas al III milenio que alcanzaran un cierto nivel de intensificación económica, fue la metalurgia, sin lugar a dudas, la actividad que desencadenó una transformación completa en las estructuras productivas en todo el Suroeste de la península ibérica a partir de los inicios del III milenio a.n.e., favoreciendo el desarrollo de varias tendencias productivas que se consolidaron con el soporte de un modelo de progresiva complejidad social y política. En los grandes centros de poder del Sur de la península ibérica, la actividad metalúrgica alcanzó un nivel de complejidad, intensidad y especialización que acabó estimulando el crecimiento de otros sectores productivos, como los vinculados al trabajo de la piedra, del hueso-marfil, del textil o, como se ha detallado en este trabajo, el alfarero.

\section{Agradecimientos}

Este trabajo ha sido desarrollado en el marco de los proyectos de investigación PIGMALION HAR-21016210 (Ministerio de Ciencia e Innovación) y HAR 2017-82966-P (Ministerio de Economía, Industria y Competitividad. Además, quisiéramos agradecer especialmente a D. Gonzalo Queipo de Llano y D. Daniel Lara Montero, responsables de la intervención arqueológica en el sector de Nueva Valencina, y a D. Juan Manuel Vargas Jiménez, arqueólogo municipal del Excmo. Ayuntamiento de Valencina de la Concepción, la constante y eficiente colaboración prestada, así como la cesión de materiales y registros arqueológicos para su estudio. Los agradecimientos son igualmente extensibles a D. Juan Carlos Mejías por el asesoramiento en materia de cartografía de Valencina de la Concepción.

\section{BIBLIOGRAFÍA}

Alchian, A.A. y Allen, W.R. (1969): Exchange and Production Theory in Use. Belmont, Wadsworth Publishing Company.

Aranda, G. (2004): "Craft specialization in pottery production during the Bronze Age in south-eastern Iberia”. Journal of Iberian Archaeology 6: 157-179.

Aranda, G. (2010): "Entre la tradición y la innovación: el proceso de especialización en la producción 
cerámica argárica”. Menga. Revista de Prehistoria de Andalucía 1: 77-95.

Arnold, D.E. (2000): "Does the standardization of ceramic pastes really mean specialization?". Journal of Archaeological Method and Theory 7: 333-375. https://doi.org/10.1023/A:1026570906712

Arnold, D.E. y Nieves, A.L. (1992): "Factors Affecting Ceramic Standardization”, en G. Bey y C. Pool (eds.), Ceramic Production and Distribution: 93113. Oxford, Westview Press, Routledge.

Arribas, A. y Molina, F. (1979): "Nuevas aportaciones al inicio de la metalurgia en la Península Ibérica. El poblado de los Castillejos de Montefrío (Granada)", en Ryan M. (ed.), The origins of metallurgy in Atlantic Europe: Proceeding of the Fifth Atlantic Colloquium: 7-34. Dublín, Stationery Office.

Arteaga, O. y Roos, A.Ma . (1995): "El Proyecto Geoarqueológico de las Marismas del Guadalquivir. Perspectivas arqueológicas de la campaña de 1992". Anuario Arqueológico de Andalucía 1992, vol. II: 329-339. Sevilla, Junta de Andalucía.

Arteaga, O.; Barragán, D.; Roos, A.M y Schulz, H.D. (2016): "El proyecto geoarqueológico puerto de Itálica”. RAMPAS: Revista Atlántica-Mediterránea de Prehistoria y Arqueología Social 18: 75-109. http:// dx.doi.org/10.25267/Rev_atl-mediterr_prehist_arqueol soc.2016.i18.08

Barragán, D. (2016): La Transgresión Flandriense en la Vega de Sevilla. El paleoestuario del río Guadalquivir. Tesis Doctoral, Universidad de Sevilla. Inédita.

Bates, D. G. y Lees, S. H. (1977): "The Role of Exchange in Productive Specialization". American Anthropologist 79: 824-841. https://doi. org/10.1525/aa.1977.79.4.02a00040

Benco, N. (1987): The Early Medieval Pottery Industry at al-Basra, Morocco. BAR International Series 341. Oxford, Archaeopress.

Benco, N. (1988): "Morphological Standardization: An Approach to the Study of Craft Specialization", en C. Kolb y L. Lackey (eds.), A Pot for All Reasons: Ceramic Ecology Revisited: 57-71. Temple, Laboratory of Anthropology, Temple University.

Blackman, M. J.; Stein, G. J. y Vandiver, P. B. (1993): "The Standardization Hypothesis and Ceramic Mass Production: Technological, Compositional, and Metric Indices of Craft Specialization at Tell Leilan, Syria". American Antiquity 58: 60-80. https://doi.org/10.2307/281454.

Borja, F. (2013): "La desembocadura del Guadalquivir en la segunda mitad del Holoceno. Síntesis paleogeográfica”, en L. García Sanjuán, J.M. Vargas,
V. Hurtado, T. Ruiz, y R. Cruz-Auñón, (eds.), El asentamiento prehistórico de Valencina de la Concepción (Sevilla). Investigación y Tutela en el 150 aniversario del descubrimiento de La Pastora: 93112. Sevilla, Universidad de Sevilla.

Brumfiel, E. y Earle, T. (eds.) (1987a): Specialization, Exchange, and Complex Societies. Cambridge, Cambridge University Press.

Brumfiel, E. y Earle, T. (1987b): "Specialization, Exchange, and Complex Societies: An Introduction", en E. Brumfiel y T. Earle (eds.), Specialization, Exchange, and Complex Societies: 1-9. Cambridge, Cambridge University Press.

Buxeda i Garrigós, J. (1999): “Alteration and contamination of archaeological ceramics: the pertubation problem". Journal of Archaeological Science 26: 295-313. https://doi.org/10.1006/jasc. 1998.0390

Caro, A. (1989): “Consideraciones sobre el Bronce Antiguo y Pleno", en M.E. Aubet, (coord.), Tartesos, Arqueología, Protohistórica del Bajo Guadalquivir: 85-97. Sabadell, Editorial AUSA.

Carriazo, J.M. (1962): "El dolmen de Ontiveros (Valencina de la Concepción, Sevilla)", en Homenaje al Profesor Cayetano de Mergelina: 209-229. Murcia, Universidad de Murcia.

Castro, P. V.; Gili, S.; Lull, V.; Mico, R.; Rihuete, C. y Risch, R. (1998): “Teoría de la producción de la vida social. Mecanismos de explotación en el sudeste ibérico". Boletín de Antropología Americana 33: 25-77. http://www.jstor.org/stable/40978127

Clark, J. (1995): “Craft Specialization as an Archaeological Category". Research in Economic Anthropology 14: 267-294.

Clark, J. y Parry, W.J. (1990): “Craft specialization and cultural complexity". Research in Economic Anthropology 12: 289-346.

Cobb, C. (2000): From Quarry to Cornfield. The Political Economy of Mississipian Hoe Productions. Alabama, The University Alabama Press.

Collantes de Terán, F. (1969): "El Dolmen de Matarrubilla”, en VV.AA. (eds.), Actas del V Symposium Internacional de Prehistoria Peninsular. Tartessos y sus Problemas: 47-61. Jerez de la Frontera (1968), Barcelona, Universidad de Barcelona.

Colomer, E. (2005): “Cerámica prehistórica y trabajo femenino en el Argar: una aproximación desde el estudio de la tecnología cerámica", en M. Sánchez Romero (ed.), Arqueología y Género: 177-219. Granada, Universidad de Granada.

Costa Caramé, M.E.; Díaz-Zorita Bonilla, M.; García Sanjuán, L. y Wheatley, D.W. (2010): “El 
asentamiento de la Edad del Cobre de Valencina de la Concepción (Sevilla). Demografía, metalurgia y organización espacial". Trabajos de Prehistoria 67(1): 87-118. https://doi.org/10.3989/ tp.2010.10032 .

Costin, C. L. y Hagstrum, M. B. (1995): "Standarization labor investment, skill, and the organization of ceramic production in Late Prehispanic Highland Peru". American Antiquity 60: 619-639. https://doi. org/10.2307/282046 .

Costin, C.L. (1991): “Craft Specialization: Issues in defining, documenting, and explaining the organization of production", en M. B. Schiffer (ed.): Archaeological methods and theory: 1-56. Tucson, The University of Arizona.

Costin, C.L. (2000): "The use of ethnoarchaeology for the archaeological study of ceramic production". Journal of Archaeological Method and Theory 7: 377-403. http://www.jstor.org/stable/20177427

Costin, C.L. (2001): “Craft Production Systems", en G. M. Feinman y T. D. Price (eds.), Archaeology at the Millennium: A Sourcebook: 273-238. New York, Springer.

Costin, C.L. (2005): “Craft Production”, en H. Maschner (ed.), Handbook of Methods in Archaeology: 1032-1105. Maryland, Altamira Press.

Cross, J.R. (1993): "Craft Specialization in Nonstratified Societies". Research in Economic Anthropology 14: 61-84.

DeMarrais, E., Castillo, L. J. y Earle, T. (1996): “Ideology, Materialization, and Power Strategies". Current Anthropology 37: 15-31. http://www.jstor.org/ stable/2744153.

Feinman, G., Kowalewski, S.A. y Blanton, R.E. (1984): "Modelling Ceramic Production and Organizational Change in the Pre-Hispanic Valley of Oaxaca, Mexico", en S.E. Van der Leeuw y A.C. Pritchard (eds.), The Many Dimension of Pottery. Ceramics in Archaeology and Anthropology: 295-337. Amsterdam, University of Amsterdam.

Fernández Gómez, F. y Oliva, D. (1985): “Excavación en el yacimiento calcolítico de Valencina de la Concepción (Sevilla). El Corte C (La Perrera)". Noticiario Arqueológico Hispano 25: 7-131.

Fernández Gómez, F. y Oliva, D. (1986): "Valencina de la Concepción (Sevilla). Excavaciones de urgencia". Revista de Arqueología 58: 19-33.

Fernández Gómez, F. y Alonso, J. (1985): “Un fondo de cabaña campaniforme en la Universidad Laboral de Sevilla". Noticiario Arqueológico Hispánico 22: 7-26.
Flannery, K.V. (1968): "The Olmec and the Valley of Oaxaca: A Model for inter-regional interaction in Formative Times", en E.P. Benson (ed.), Dumbarton Oaks Conference on the Olmec: 79-110. Cambridge, Harvard University.

Flannery, K.V. (1972): “Cultural Evolution of Civilization". Annual Review of Ecology and Systematics 3: 399-426. http://www.jstor.org/stable/2096853

Galán, E. y Pérez, J. L. (1989): Geología de Sevilla y alrededores y características geotécnicas de los suelos del área urbana. Sevilla, Ayuntamiento de Sevilla.

García Sanjuán, L. (2013): “El asentamiento de la Edad del Cobre de Valencina de la Concepción: estado actual de la investigación, debates y perspectivas", en L. García Sanjuán, J.M. Vargas, V. Hurtado, T. Ruiz y R. Cruz-Auñón (eds.), El asentamiento prehistórico de Valencina de la Concepción (Sevilla). Investigación y Tutela en el 150 aniversario del descubrimiento de La Pastora: 21-59. Sevilla, Universidad de Sevilla,.

García Sanjuán, L. y Murillo-Barroso, M. (2013): "Social Complexity in Copper Age Southern Iberia (ca. 3200-2200 Cal BC). Reviewing the "State" Hypothesis at Valencina de la Concepción (Seville, Spain)", en M. Cruz Berrocal, L. García Sanjuán y A. Gilman (eds.): The Prehistory of Iberia. Debating Early Social Stratification and the State: 119140. London, Routledge.

García-Sanjuán, L., Vargas, J.M., Hurtado, V., Ruiz, T. y Cruz-Auñón, R. (2013): El asentamiento prehistórico de Valencina de la Concepción (Sevilla). Investigación y Tutela en el 150 aniversario del descubrimiento de La Pastora. Sevilla, Universidad de Sevilla.

García-Sanjuán, L., Luciañez Triviño, M., Schuhmacher, T. X., Wheatley, D. y Banerjee, A. (2013): "Ivory Craftsmanship, Trade and Social Significance in the Southern Iberian Copper Age: The Evidence from the PP4-Montelirio Sector of Valencina de la Concepción (Seville, Spain)". European Journal of Archaeology 16(4): 610-635. https://doi.org/ 10.1179/1461957113Y.0000000037.

García Sanjuán, L., Scarre, C. y Wheatley, D.W. (2017): "The Mega-Site of Valencina de la Concepción (Seville, Spain): Debating Settlement Form, Monumentality and Aggregation in Southern Iberian Copper Age Societies". Journal World Prehistory 30: 239-257. https://doi.org/10.1007/s10963017-9107-6 .

González-Vílchez, M. C. (1982): Estudio Físico-Químico y Tecnológico de una Serie de Productos Cerámicos del Yacimiento Arqueológico de Valencina 
de la Concepción (Sevilla). Tesis Doctoral, Universidad de Sevilla. Inédita.

González-Vílchez, M.C., García Ramos, G., González García, F., González Rodríguez, M. y Fernández Gómez, F. (1999): "Estudio arqueométrico de un conjunto de piezas cerámicas del yacimiento arqueológico de Valencina de la Concepción (Sevi1la)", en J. Capel (ed.), Arqueometría y Arqueología: 69-76. Granada, Universidad de Granada.

Hein, A., Mommsen, H. y Maran, J. (1999): “Element concentration distributions and most discriminating elements for provenancing by neutron activation analyses of ceramics from Bronze Age Sites in Greece". Journal of Archaeological Science 26: 1053-1058. https://doi.org/10.1006/ jasc.1999.0423.

Inácio, N. (2015): Alfarería y Metalurgia. Contribución del análisis arqueométrico para el estudio de los patrones de producción, distribución y consumo de cerámica en el Suroeste de la Península Ibérica durante el III Milenio A.N.E. Tesis Doctoral, Universidad de Huelva. Inédita.

Inácio, N., Nocete, F., Aldana, P., Pajuelo, A. y Bayona, M.R. (2017): "Producción y consumo de cerámica campaniforme en Valencina de la Concepción: Una propuesta interpretativa desde el análisis de los contextos de la Calle Trabajadores", en V. Gonçalves, (eds.), Sinos e Vasos. Junto ao Oceano e mais longe. Aspectos da presença campaniforme na Península Ibérica: 228-241. Lisboa, UNIARQ.

Lara, D.A., Barragán, D. y Garrido, M. (2006): “El asentamiento calcolítico del Parque de Miraflores: resultados preliminares". Spal 13: 245-256. https:// doi.org/10.12795/spal.2004.i13.09 .

Longacre, W.A. (1999): "Standardization and Specialization: What's the Link?", en J. M. Skibo y G. M. Feinman (eds.), Pottery and People: A Dynamic Interaction: 44-58. Salt Lake City, University of Utah Press.

Martín de la Cruz, J.C. y Miranda, J.M. (1988): “El poblado calcolítico de Valencina de la Concepción (Sevilla): Una revisión crítica". Cuadernos de Prehistoria y Arqueología Universidad Autónoma de Madrid 15: 37-67. http://hdl.handle.net/10486/639.

Marx, K. (1990): O Capital. Crítica da Economía Política. Lisboa, Edições Progresso-Editorial Avante.

Nocete, F. (2001): Tercer Milenio antes de Nuestra Era. Relaciones y contradicciones centro/periferia en el Valle del Guadalquivir. Barcelona, Bellaterra.

Nocete, F. (2014): "Las sociedades complejas (IV y III Milenio cal B.C.) en la Iberia Meridional", en M. Almagro-Gorbea (ed.), Protohistoria de la Península
Ibérica: Del Neolítico a la Romanización: 83-94. Burgos, Fundación Atapuerca-Universidad de Burgos.

Nocete, F., Sáez, R., Bayona, M.R., Nieto, J.M., Peramo, A., López, P., Gil-Ibarguchi, J.I., Inácio, N., García y S., Rodríguez (2014): “Gold in the Southwest of the Iberian Peninsula during the 3rd Millenium BC". Journal of Archaeological Science 41: 691704. https://doi.org/10.1016/j.jas.2013.10.006 .

Nocete, F., Sáez, R., Bayona, M.R., Peramo, A., Inacio, N. y Abril, D. (2011): "Direct chronometry (14C AMS) of the earliest copper metallurgy in the Guadalquivir Basin (Spain) during the Third millennium BC: First Regional Database". Journal of Archaeological Science 38: 3278-3295. https://doi. org/10.1016/j.jas.2011.07.008 .

Nocete, F., Vargas, J.M., Schuhmacher, T.X., Banerjee, A. y Dindorf, W. (2013): "The ivory workshop of Valencina de la Concepción (Seville, Spain) and the identification of ivory from Asian elephant on the Iberian Peninsula in the first half of the 3rd millenium BC". Journal of Archaeological Sicience 40(3): 1579-1592. https://doi.org/10.1016/j. jas.2012.10.028.

Nocete, F.; Queipo, G.; Sáez, R.; Nieto, J.M.; Inácio, N.; Bayona, M.R.; Peramo, A.; Vargas, J.M.; Cruz, R.; Gil-Ibarbucci, J.I. y Santos, J.F. (2008): “The smelting quarter of Valencina de la Concepción (Seville, Spain): the specialised copper industry in a political centre of the Guadalquivir Valley during the Third millennium BC (2750-2500 BC)". Journal of Archaeological Science 35: 717-732. https:// doi.org/10.1016/j.jas.2007.05.019 .

Obermaier, H. (1919): "El Dolmen de Matarrubilla (Sevilla)". Memoria de la Comisión de Investigaciones Paleontológicas y Prehistóricas 26. Madrid, Museo de Ciencias Naturales.

Orihuela, A. (1999): Historia de la Prehistoria: el Suroeste de la Península Ibérica. Huelva, Diputación Provincial de Huelva..

Pajuelo, A. y López Aldana, P.M. (2001): “Ideología y control político durante el III Milenio a.n.e. en el Bajo Guadalquivir". Revista Atlántica-Mediterránea de Prehistoria y Arqueología Social IV: 229255. http://dx.doi.org/10.25267/Rev_atl-mediterr_ prehist_arqueol_soc.2001.v4.08.

Pellicer, M. (1979): “La prehistoria de Andalucía Occidental como función geográfica". XV Congreso $\mathrm{Na}$ cional de Arqueología: 25-30. Zaragoza.

Peregrine, P. (1991): "Some Political Aspects of Craft Specialization". World Archaeology 23: 1-11. http://www.jstor.org/stable/124725 . 
Rice, P. (1981): "Evolution of specialized pottery production: A trial model". Current Anthropology 22(3): 19-40. http://www.jstor.org/stable/2742199.

Rice, P. (1987): Pottery Analysis, A Sourcebook. Chicago, University of Chicago Press.

Rice, P. (1991): "Specialization, Standardization and Diversity: A Retrospective", en R.L. Bishop y F.W. Lange (eds.), The Ceramic Legacy of Anna O. Sheppard: 257-279. Boulder, University Press of Colorado.

Rosa, J. y Castro, A. (2004): "Magmatismo de la Zona Sudportuguesa”. En J.A. Vera (ed.): Geología de España: 215-222. Madrid, SGE-IGME.

Rosa, J. (1992): Petrología de las rocas básicas y granitóides del batolito de la Sierra Norte de Sevilla. Tesis Doctoral, Universidad Sevilla, Sevilla. Inédita.

Ruiz Mata, D. (1975a): “Cerámicas del Bronce del poblado de Valencina de la Concepción (Sevilla)". Madrider Mitteilungen 16: 80-110.

Ruiz Mata, D. (1975b): “Cerámicas del Bronce del poblado de Valencina de la Concepción (Sevilla): los platos". Cuadernos de Prehistoria y Arqueología de la Universidad Autónoma de Madrid 2: 123-149. http://dx.doi.org/10.15366/cupauam1975.2.010 .

Ruiz Mata, D. (1983): "El yacimiento de la Edad del Bronce de Valencina de la Concepción (Sevilla) en el marco cultural del Bajo Guadalquivir". I Congreso de Historia de Andalucía: 183-208. Córdoba (1976), Córdoba.

Sáez, R., Almodóvar, G.R. y Pascual, E. (1996): "Geological constrait on massive sulphide genesis in the Iberian Pyrite Belt". Ore Geological Review 11: 429-451. https://doi.org/10.1016/S0169_ 1368(96)00012-1.

Sassaman, K. E. (1998): “Crafting Cultural Identity in Hunter-Gatherer Economies", en C. Costin y R. Wright (eds.), Craft and Social Identity, Archeological Papers No. 8: 93-108. Arlington, American Anthropological Association.

Silva, C.T. y Soares, J. (1977): “Contribuição para o conhecimento dos povoados calcolíticos do Baixo Alentejo e Algarve". Setúbal Arqueológica 2-3: 179-272.

Silva, C.T. y Soares, J. (1981): Pré-História da área de Sines. Lisboa, Gabinete da área de Sines.

Sinopoli, C. (1988): "The Organization of Craft Production at Vijayanagara, South India". American Anthropologist 90: 580-597. https://doi. org/10.1525/aa.1988.90.3.02a00040 .

Stark, M.T. (1995): "Economic intensification and ceramic specialization in the Philippines: A view from Kalinga". Research in Economic Anthropology 16: 179-226.
Stein, G.J. (1996): "Producers, Patrons, and Prestige: Craft Specialists and Emergent Élites in Mesopotamia from 5500-3100 B.C", en Craft B. Wailes (ed.), Specialization and Social Evolution: In Memory of V. Gordon Childe: 25-38. Philadelphia, University of Pennsylvania Museum.

Stein, G.J. (1998): "Heterogeneity, Power, and Political Economy: Some Current Research Issues in the Archaeology of Old World Complex Societies". Journal of Archaeological Research 6: 1-44. https://doi. org/10.1023/A:1022801712684.

Stein, G.J. y Blackman, J.M. (1993): “The Organizational Context of Specialized Craft Production in Early Mesopotamian States". Research in Economic Anthropology 14: 29-60.

Tornos, F. (2008): "La geología y metalogenia de la Faja Pirítica Ibérica". MACLA Revista de la Sociedad Española de Mineralogía 10: 13-23.

Tosi, M., (1984): “The Notion of Craft Specialization and Its Representations in the Archaeological Record of Early States in the Turanian Basin", en M. Spriggs (ed.), Marxist Perspectives in Archaeology: 22-52. Cambridge, Cambridge University Press.

Tsolakidou, A., Kilikoglou, V., Kiriatzi, E. y Day, P.M. (2002): "Investigating Petrological and Chemical Grouping of Early Minoan Cooking Vessels", en V. Kilikoglou, A. Hein e Y. Maniatis (eds.), Modern Trends in Scientific Studies on Ancient Ceramics, BAR International Series 1011: 19-29. Oxford, Archaeopress.

Tubino, F.M. (1868): "Monumentos prehistóricos de Castilleja de Guzmán”. Estudios prehistóricos: 49-58.

Vargas, J.M. (2004): Carta Arqueológica Municipal de Valencina de la Concepción. Monografías de Arqueología. Sevilla, Consejería de Cultura, Junta de Andalucía.

Vargas, J.M. (2007): Memoria final de la Actuación Arqueológica Preventiva en la Parcela del nuevo Instituto de Enseñanza Secundaria de Valencina de la Concepción (Sevilla). Junta de Andalucía (Consejería de Cultura). Sevilla. Inédito.

Vargas, J.M., Nocete, F. y Ortega, M. (2010): “Excavaciones arqueológicas en la parcela del nuevo IES de Valencina de la Concepción (Sevilla)". Anuario Arqueológico de Andalucía 2005: 3340-3352.

Whitbread, I. K. (1995): Greek Transport Amphorae: A Petrological and Archaeological Study. Fitch Laboratory Occasional Paper, 4. Atenas, British School at Athens. 